\title{
Carrying simplices for competitive maps
}

\author{
Stephen Baigent
}

\begin{abstract}
The carrying simplex is a finite-dimensional, attracting Lipschitz invariant manifold that is commonly found in both continuous and discrete-time competition models from Ecology. It can be studied using the graph transform and cone conditions often applied to study attractors in continuous-time finite and infinitedimensional models from applied mathematics, including chemical reaction networks and reaction diffusion equations. Here we show that the carrying simplex can also be studied from the point of view of the graph transform and cone conditions. However, unlike many of the models mentioned above, we do not use - at least directly - a gap condition that is often used to establish existence of a globally and exponentially attracting manifold. Instead we use contraction of phase volume to 'suck' hypersurfaces together uniformly, and ultimately onto the carrying simplex. We give a proof of the existence of the carrying simplex for a class of competitive maps, viewed here as also normally monotone maps. The result is not new, but is carried out in the framework of the graph transform to indicate how the carrying simplex relates to other well-known classes of invariant manifolds. We also discuss the relation between hypersurfaces with positive normals, unordered hypersurfaces and also the type of maps that preserve these types of hypersurfaces. Finally we review several examples from models in Ecology where the carrying simplex is known to exist.
\end{abstract}

\section{Introduction}

Let us start by considering 1-dimensional difference equations of the form $x_{n+1}=$ $F\left(x_{n}\right)=x_{n} f\left(x_{n}\right)$ on the nonnegative reals $\mathbb{R}_{+}=[0, \infty)$, where $f: \mathbb{R}_{+} \rightarrow \mathbb{R}_{+}$is at least continuous and positive on $\mathbb{R}_{+}$. We recall that the map $F: \mathbb{R}_{+} \rightarrow \mathbb{R}_{+}$satisfies

Stephen Baigent

Department of Mathematics, UCL, Gower Street, London WC1E 6BT. e-mail: steve.baigent@ucl.ac.uk 
$x_{n}=F^{n}\left(x_{0}\right)$ where $F^{n}$ is the composition of $F$ with itself $n$ times and $x_{0}$ is an initial point. The study of the difference equation $x_{n+1}=F\left(x_{n}\right)$ is equivalent to the study of the repeated application of $F$, and we take the latter viewpoint here.

As our first example, consider the Beverton-Holt map from Ecology:

$$
F(x)=x f(x), f(x)=\frac{r}{1+a x}, r, a>0 .
$$

Clearly $\mathbb{R}_{+}$is invariant under $F$, and $F \geq 0$ is an increasing function bounded above by $\frac{r}{a}$. Moreover $f$ is strictly positive on $\mathbb{R}_{+} . F$ has the positive fixed point $x^{*}=\frac{r-1}{a}$ whenever $r>1$. When it exists, $x^{*}$ globally and asymptotically attracts $\mathbb{R}_{+}$bar the origin, and the origin is an unstable fixed point. When $r \leq 1$ the origin is the unique globally attracting fixed point. The positive fixed point $x^{*}=\frac{r-1}{a}(r>1)$ is known as the carrying capacity, as it is the maximum steady population size that can be stably supported by the habitat. Notice that $F$ is invertible on $\mathbb{R}_{+}$and the derivative of its inverse is positive.
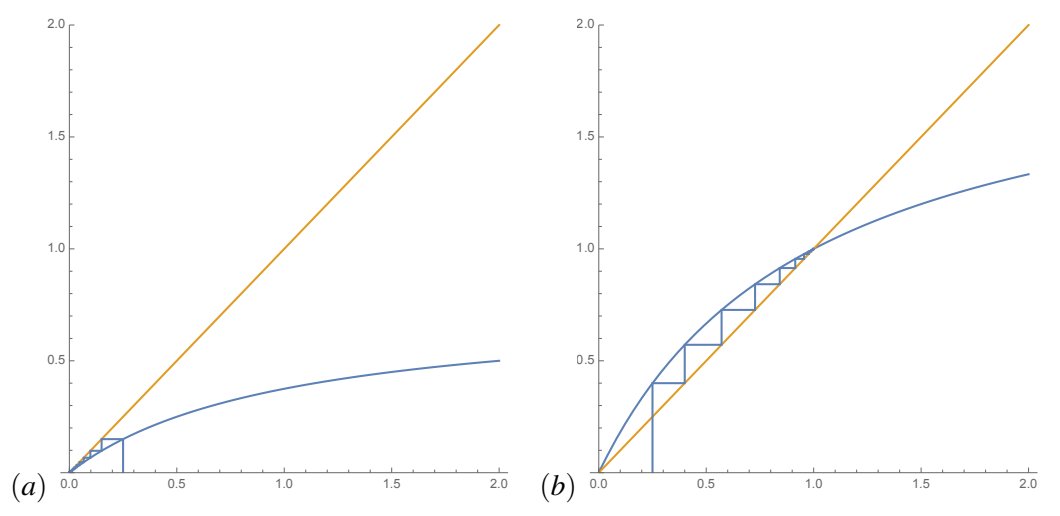

Fig. 1 Beverton-Holt model (1). (a) $r=0.75, a=1$. (b) $r=2, a=1$. When $r \leq 1$ there is no positive fixed point and 0 is globally attracting. For $r>1$ there is a unique positive fixed point $x^{*}=\frac{r-1}{a}$ which attracts $\mathbb{R}_{+} \backslash\{0\}$. In (b) the positive fixed point is known as the carrying capacity. The map $F$ is one-to-one from its domain $\mathbb{R}_{+}$onto its compact image $F([0, \infty))=\left[0, \frac{r}{a}\right]$.

Another well-known model is the Ricker model, again from Ecology:

$$
F(x)=x f(x), f(x)=e^{r(1-a x)}, r, a>0 .
$$

This map can generate some very complicated behaviour, including chaos (when $r>2$ ). As with the Beverton-Holt map, $F$ maps $\mathbb{R}_{+}$into itself, and $f$ is positive on $\mathbb{R}_{+}$. However unlike the Beverton-Holt model, now $F$ is not everywhere increasing. Here $F^{\prime}(x)=e^{r(1-a x)}(1-r a x)$, so that $F^{\prime}$ changes sign at $(r a)^{-1}$ and $F$ is only increasing in $\left[0,(r a)^{-1}\right], F$ restricted to $\left[0,(r a)^{-1}\right]$ is invertible. In fact, for each $y \in F((0, \infty))$ the cardinality of $F^{-1}(y)$ is 2 (counting $x=(r a)^{-1}$ twice). There is 
always an interior fixed point at $x^{*}=1 / a$ which is the carrying capacity, but it may not be globally attracting. When $r<1$ the fixed point $1 / a$ lies to the left of $(r a)^{-1}$ and so is within the interval $\left[0,(r a)^{-1}\right]$ where $F$ is invertible. When $r>1$, the fixed point lies outside the interval where $F$ is invertible. Note that $F^{\prime}(1 / a)=1-r$, so that $x^{*}=1 / a$ is unstable for $r>2$. But for $1<r \leq 2, x^{*}=1 / a$ is globally asymptotically stable (bar the origin). For $1<r \leq 2, x^{*}=1 / a$ is the carrying capacity. See Figure 2 for illustrations.
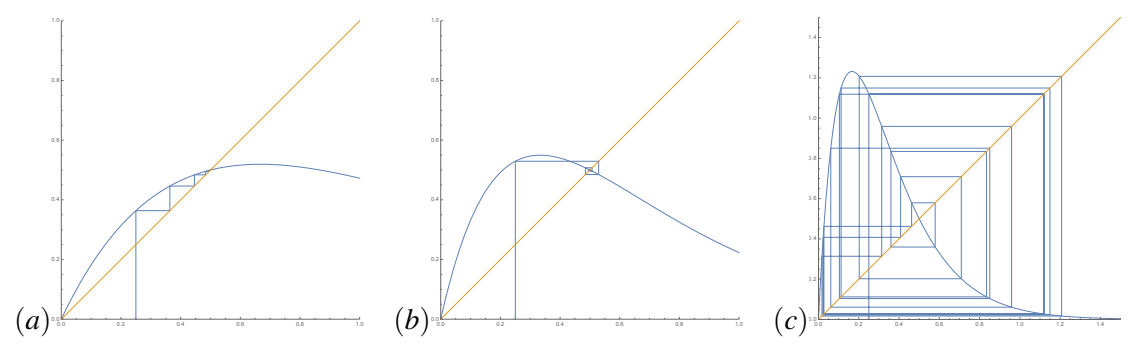

Fig. 2 Ricker model (2). (a) $r=0.75, a=2$. (b) $r=1.5, a=2$, (c) $r=3, a=2$. In (a) $F$ is invertible up to $x=2 / 3, x^{*}=1 / 2$ is globally asymptotically stable on $(0, \infty)$ and lies to the left of $x=2 / 3$. For (b), $F$ is invertible up to $x=1 / 3$ which now lies to the left of $x^{*}=1 / 2$, which remains globally asymptotically stable on $(0, \infty)$. Finally in (c) $F$ is invertible up to $x=1 / 6$, but now $x^{*}=1 / 2$ is unstable, and the orbits appear to be chaotic.

In two dimensions, let us consider the planar Leslie-Gower map, again from Ecology. The densities of two interacting species are $x_{1}, x_{2}$ and $r_{1}, r_{2}, a, b$ are positive parameters, and the Leslie-Gower map is the differentiable map $F$ of the first quadrant $C_{+}$into itself defined by

$$
F(x)=\left(\frac{r_{1} x_{1}}{1+x_{1}+a_{12} x_{2}}, \frac{r_{2} x_{2}}{1+y_{1}+a_{21} x_{1}}\right), \quad x=\left(x_{1}, x_{2}\right) \in \mathbb{R}_{+}^{2} .
$$

Restricted to either of the invariant positive axes, the map becomes the 1 -dimensional Beverton-Holt map. Cushing et al. [11] (see also [36]) showed that if (a) $r_{1}, r_{2}<1$ then $e_{0}=(0,0)$ is globally asymptotically stable on $\mathbb{R}_{+}^{2}$, (b) $r_{1}>1, r_{2}<1$ then $q_{1}=\left(r_{1}-1,0\right)$ is globally asymptotically stable on int $\mathbb{R}_{+}^{2}$, (c) $r_{1}<1, r_{2}>1$ then $q_{2}=\left(0, r_{2}-1\right)$ is globally asymptotically stable on int $\mathbb{R}_{+}^{2}$. When $r_{1}>1, r_{2}>1, e_{0}$ is a repeller and there are 4 distinct cases: When (a) $a_{21}\left(r_{1}-1\right)>r_{2}-1, r_{1}-1>$ $a_{12}\left(r_{2}-1\right)$ then $q_{1}$ is asymptotically stable on int $\mathbb{R}_{+}^{2}$ and $q_{2}$ is a saddle, (b) $a_{21}\left(r_{1}-1\right)<r_{2}-1, r_{1}-1<a_{12}\left(r_{2}-1\right)$ then $q_{2}$ is asymptotically stable on int $\mathbb{R}_{+}^{2}$ and $q_{1}$ is a saddle, (c) when $a_{21}\left(r_{1}-1\right)<r_{2}-1, r_{1}-1>a_{12}\left(r_{2}-1\right)$ then the interior fixed point $p$ is globally asymptotically stable on int $\mathbb{R}_{+}^{2}$ and (d) when $a_{21}\left(r_{1}-1\right)>r_{2}-1, r_{1}-1<a_{12}\left(r_{2}-1\right)$ then the interior fixed point $p$ is a saddle. Here we are concerned with the case $r_{1}, r_{2}>1$. An interior fixed point has coordi- 
nates $p=\left(\frac{a_{12}\left(1-r_{2}\right)+r_{1}-1}{1-a_{12} a_{21}}, \frac{r_{2}-1+a_{21}\left(1-r_{1}\right)}{1-a_{12} a_{21}}\right)$ and so is feasible, since we are assuming $r_{1}>1, r_{2}>1$ when either $a_{12}<\frac{r_{1}-1}{r_{2}-1}<\frac{1}{a_{21}}$ or $\frac{1}{a_{21}}<\frac{r_{1}-1}{r_{2}-1}<a_{12}$.

In Figure 3 we show two examples of orbits for the planar Leslie-Gower model. In (a) $r_{1}=r_{2}=2, a_{12}=2, a_{21}=3$, so that there is an interior fixed point that is an unstable saddle, and in (b) $r_{1}=r_{2}=2, a_{12}=1 / 2, a_{21}=1 / 3$ there is an interior fixed point that attracts int $\mathbb{R}_{+}^{2}$. Also shown in each of (a), (b) is a decreasing invariant curve, which we denote by $\Sigma$, that joins the axial fixed points $q_{1}=(1,0)$ and $q_{2}=$ $(0,1)$. We see that all orbits are attracted to $\Sigma$ apart from the origin. $\Sigma$ is known as the carrying simplex for each example, and is an analogue for the carrying capacity in the previous two one-dimensional models discussed.

Carrying simplices were first introduced, though not by that name, for continuous time competitive systems [18, 41], and later for maps in [12, 35, 33]. They offer a generalisation of the idea of the carrying capacity, defined in a multispecies model for each species as the maximum stable population that a species can attain in the absence of all others, to a state of balance that involves all species present. Note that the carrying capacity of a species in a multispecies model is just the nonzero axial fixed point, if it exists.

(As the story for the planar Ricker model is more complicated, we will postpone looking at its carrying simplex till subsection 5.2.)

(a)

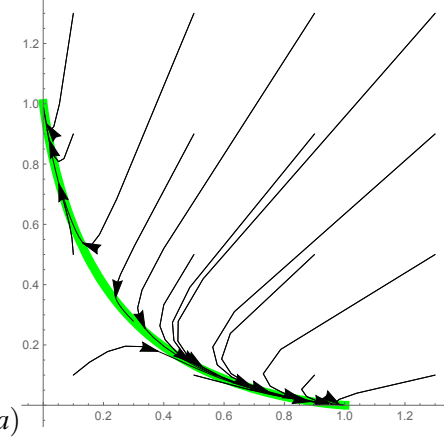

(b)

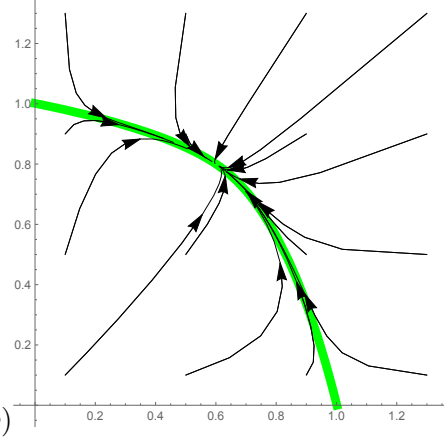

Fig. 3 Carrying simplices for the Leslie-Gower model (3). (a) Concave carrying simplex for $r_{1}=$ $r_{2}=2, a_{12}=2, a_{21}=3$. (b) Convex carrying simplex $r_{1}=r_{2}=2, a=a_{12}, a_{21}=1 / 3$.

Hirsch provided the following definition for the carrying simplex. Let $C_{+}=$ $[0, \infty)^{d}$ and $F: C_{+} \rightarrow C_{+}$be a map. (Here when we say $f$ is a map, this assumes that $f$ is at least continuous).

Definition 1 (Carrying Simplex [19]). A carrying simplex $\Sigma \subset C_{+} \backslash\{0\}$ is a set with the following properties:

CS1 $\Sigma$ is compact and invariant. 
CS2 For every $x \in C_{+} \backslash\{0\}$ the trajectory of $x$ is asymptotic to the trajectory of some $y \in \Sigma: \lim _{n \rightarrow \infty}\left|F^{n}(x)-F^{n}(y)\right|=0$.

CS3 $\Sigma$ is unordered (no two points can be ordered componentwise).

Remark 1. It is the last property CS3 of being an unordered manifold that distinguishes the carrying simplex from other compact invariant hypersurfaces.

Remark 2. CS2 is stronger than saying that $\Sigma$ is globally attracting, and is known in the literature as asymptotic completeness (e.g. in the context of inertial manifolds). Asymptotic completeness says that for any full orbit there is an orbit in $\Sigma$ that shadows the full orbit for all time, and becoming progressively closer.

From Figure 3, it is clear that for this Leslie-Gower model $\Sigma$ is compact and invariant, and CS2 is satisfied. For the last requirement, unordered means that no two points $x=\left(x_{1}, x_{2}\right)$ and $y=\left(x_{1}, y_{2}\right)$ satisfy $x-y \in \mathbb{R}_{+}^{2}$ or $y-x \in \mathbb{R}_{+}^{2}$, and this is satisfied because $\Sigma$ is the graph of a (strictly) decreasing function. An alternative description of $\Sigma$ for this model is that it is both the boundary (relative to $\mathbb{R}_{+}^{2}$ ) of the basin of repulsion of the origin and the boundary (relative to $\mathbb{R}_{+}^{2}$ ) of the basin of repulsion of infinity.

In three dimensions the carrying simplex becomes a surface. Simple examples of carrying simplices are then provided by the May-Leonard map, again from ecology. We take $x=\left(x_{1}, x_{2}, x_{3}\right) \in \mathbb{R}_{+}^{3}, \alpha, \beta>0$ and the define the May-Leonard map $F_{M L}$ : $\mathbb{R}_{+}^{3} \rightarrow \mathbb{R}_{+}^{3}$ by

$F_{M L}(x)=\left(\frac{r x_{1}}{1+x_{1}+\alpha x_{2}+\beta x_{3}}, \frac{r x_{2}}{1+x_{2}+\alpha x_{3}+\beta x_{1}}, \frac{r x_{3}}{1+x_{3}+\alpha x_{1}+\beta x_{2}}\right), r>1$.

Figure 4 shows carrying simplices for the May-Leonard model together with a

(a)

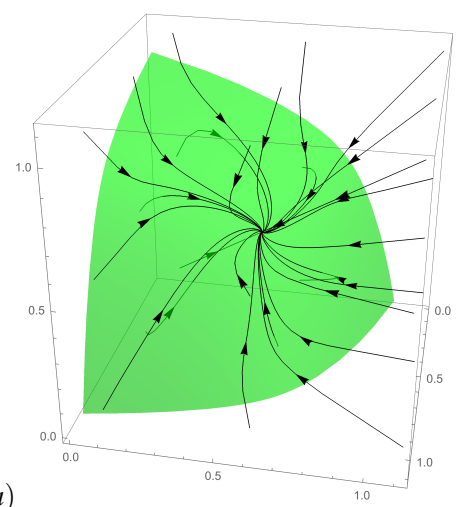

(b)

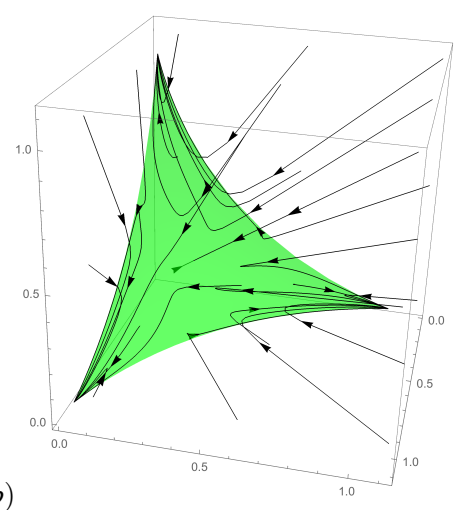

Fig. 4 Carrying simplices for the May-Leonard model (4) with $r=2$. (a) Convex carrying simplex for $\alpha=1 / 2, \beta=1 / 3$ (b) Concave carrying simplex $\alpha=2, \beta=3 / 2$ ). 
selection of orbits. In both figures it is clear that both CS1 and CS2 are satisfied. Moreover, since each surface $\Sigma$ is decreasing in each coordinate direction, $\Sigma$ is an unordered manifold, so that CS3 is satisfied.

The planar Leslie-Gower model and the 3-species May-Leonard model are examples of competitive maps, and as we shall see below, it is their competitive character that enables us to show that the carrying simplex exists and is unordered. Alternatively, we may see these models in terms of normally monotone maps in that they map hypersurfaces with nonnegative normals to hypersurfaces with nonnegative normals. We will have more to say about these concepts later. In the next section we introduce some notation and terminology associated with the study of carrying simplices and competitive maps.

\section{Preliminaries and notation}

Let $d \geq 1$ be an integer, $\mathbb{R}_{+}=[0, \infty), C_{+}=\mathbb{R}_{+}^{d}$ and int $C_{+}$denote the interior of $C_{+}$. We will consider maps $F: C_{+} \rightarrow C_{+}$that are at least continuously differentiable on a neighbourhood of $C_{+} . O^{+}(x)$ denotes the forward orbit of $F$ through $x$, i.e. $O^{+}(x)=\left\{F^{k}(x): k \in \mathbb{Z}_{+}\right\}$where $\mathbb{Z}_{+}=\{0,1, \ldots\}$ are the nonnegative integers and by $F^{k}$ we mean that map formed by composing $F$ with itself $k$ times. If $F\left(C_{+}\right)$is compact, for each $x \in C_{+}$the omega limit set $\omega(x)$ consisting of all limit points in $O^{+}(x)$ is nonempty and compact. When $\omega(x)=\{y\}$, then $y$ is a fixed point of $F$ and we say that $O^{+}(x)$ converges to $y$. We will let $e_{i} \in \mathbb{R}^{d}$ be the column vector consisting of a one at position $i$ and zeros elsewhere, and set $e=e_{1}+\cdots e_{d}, \hat{e}=$ $\frac{e}{\sqrt{d}}$ throughout. Here $\Delta_{d-1}=\left\{u \in C_{+}: \sum_{i=1}^{d} u_{i}=1\right\}$, commonly known as the unit probability simplex. The symbol $I_{d}:=\{1,2, \ldots, d\}$. We will use $M^{T}$ to denote the transpose of a matrix $M$ and $M^{-T}$ to denote $\left(M^{-1}\right)^{T}$ when the inverse $M^{-1}$ exists. We will also use $D[x]$ to denote the diagonal matrix whose diagonal elements are $x=\left(x_{1}, \ldots, x_{d}\right)$.

By $D F$ we mean the differential of $F$, i.e. the linear map of $\mathbb{R}^{d}$ into itself. $D F^{k}:=$ $D\left(F^{k}\right)$ and when the inverse of $F$ exists $D F^{-1}=D\left(F^{-1}\right)$. By contrast $(D F)^{-1}$ is the inverse of the linear map $D F$. By $\|x\|_{2}$ is meant the Euclidean norm of $x \in \mathbb{R}^{d}$.

For $x, y \in \mathbb{R}^{d}$, we write $x \leq y \Leftrightarrow y-x \in C_{+}$. As refinements we write $x<y$ if $x \leq y$ and $x \neq y$, and finally $x \ll y$ if $y-x \in \operatorname{int} C_{+}=(0, \infty)^{d}$. When $x, y \in \mathbb{R}^{d}$ and $x \leq y$, by $[x, y]$ we mean the closed order interval $\left\{z \in \mathbb{R}^{d}: x \leq z \leq y\right\}$. We say that two points $x, y$ are related $(x \sim y)$ if either $y \leq x$ or $x \leq y$.

Here we focus on competitive Kolmogorov maps where we define

Definition 2 (Kolmogorov map). A Kolmogorov map is a map $F: C_{+} \rightarrow C_{+}$that can be written componentwise as $F_{i}(x)=x_{i} f_{i}(x), i=1, \ldots, d$, where each $f_{i}$ is at least continuously differentiable on an open neighbourhood of $C_{+}$. We denote the set of Kolmogorov maps by $\mathscr{K}$. 
Kolmogorov maps leave $C_{+}$and all of its facets invariant. Ecologically this corresponds to future absence of a species that is originally absent.

Definition 3 (Hypersurface). A hypersurface $S$ is a subset of $\mathbb{R}^{d}$ such that for every point $x \in S$ there exists a neighbourhood $U_{x} \subset \mathbb{R}^{d}$ of $x$ and a continuously differentiable function $\phi: U_{x} \rightarrow \mathbb{R}$ with $D \phi \neq 0$ on $U_{x}$ such that $S \cap U_{x}=\left\{x \in U_{x}: \phi(x)=0\right\}$.

Remark 3. Note that the definition does not specify that a hypersurface $S$ is connected although here we mostly encounter connected and compact hypersurfaces.

In $[6,4,5]$ Baigent studied the geometry of carrying simplices of competitive systems and used widely that a competitive system, in continuous or discrete time, the dynamics takes hypersurfaces with nonnegative normal to hypersurfaces with nonnegative normal. As we will show below, the value of working with hypersurfaces with nonnegative normal is that these hypersurfaces are graphs of Lipschitz functions with a uniform Lipschitz constant. It is therefore useful to seek codimensionone invariant manifolds of competititive systems as limit of sequences of hypersurfaces with nonnegative normal. In their study of global stability of interior fixed points of monotone maps, Balreira, Elaydi and Luís made hypersurfaces with nonnegative normals central to their approach [8] and defined:

Definition 4 (Normally monotone map). Let $U \subseteq \mathbb{R}^{d}$ be open. We say that a map $F: U \rightarrow \mathbb{R}^{d}$ is normally monotone on $U$ if $D F$ is nonsingular and $(D F)^{-1}>0$ on $U$.

Definition 5 (Monotone region). Let $U \subseteq C_{+}$be open. We say that $U$ is a monotone region if $\partial U$ is a $C^{1}$ hypersurface such that for any $x \in \partial U \cap \operatorname{int} C_{+}$, the outward normal to $\partial U$ is nonzero and nonnegative.

As explained in [8] a monotone region is one whose boundary relative to $C_{+}$is a hypersurface with positive normal and a normally monotone map maps hypersurfaces with positive normal to hypersurfaces with positive normal.

Normally monotone maps are closely related (see lemma 3.1 [8] for equivalence for planar maps on $\mathbb{R}_{+}^{2}$-convex sets) to the more standard concepts of competitive maps (see, for example, $[16,17])$ :

Definition 6 (Competitive map). Let $U \subseteq \mathbb{R}^{d}$ be a set. We say that a map $F: U \rightarrow$ $\mathbb{R}^{d}$ is competitive if $x<y$ whenever $F(x)<F(y)$ and $x, y \in U$.

Definition 7 (Strongly competitive map). Let $U \subseteq \mathbb{R}^{d}$ be a set. $F: U \rightarrow \mathbb{R}^{d}$ is strongly competitive if $x \ll y$ whenever $F(x)<F(y)$ and $x, y \in U$.

Definition 8 (Unordered set). A set $U \subseteq \mathbb{R}^{d}$ is unordered if it contains no related points, i.e. if $x, y \in U$ and either $x \leq y$ or $y \leq x$ then $x=y$.

For example, the unit probability simplex $\Delta_{d-1}$ is an unordered manifold. For suppose $u, v \in \Delta_{d-1}$ and $u>v$. Then $\sum_{k=1}^{d} u_{i}>\sum_{k=1}^{d} v_{i}$ which is a contradiction since both sums must equal one. 
The relationship between normally monotone maps, competitive maps, unordered hypersurfaces and hypersurfaces with nonnegative normals will be discussed in section 4. Both competitive and normally monotone maps take $C^{1}$ hypersurfaces of nonnegative normal to hypersurfaces of nonnegative normal.

Also of importance here in linking normally monotone maps to competitive maps is the spectral radius of a matrix. We recall

Definition 9 (Spectral radius). The spectral radius $\rho(W)$ of a square matrix $W$ is the maximum of the modulus of the eigenvalues of $W$.

There is a growing literature on the existence of carrying simplices for competitive systems. Earlier results were for differential equations [18, 19], and later for maps (including those arising from periodic differential equations) [12, 33, 39, 22, $30,13]$. Over time, the conditions imposed to ensure the existence of the carrying simplex have been refined. In several early papers [34, 33] Smith studied the boundary of the basin of repulsion of the origin for a competitive map derived from the Poincaré map of a competitive periodic differential equation, and compared it to the boundary of the global attractor, conjecting that the two boundaries were the same. Later by introducing a new condition on the map, Wang and Jiang in [39] improved on the results of Smith [33], showing that the two boundaries were then identical. This common boundary is an unordered manifold in the sense that no two points in the manifold can be order-related. Takáč [37] shows that invariant hypersurfaces of codimension-one are common in competitive systems, in that any nonempty unordered invariant set (e.g. a fixed point) lies in an invariant unordered closed hypersurface. Takáč's results suggest that the main issue for existence of the carrying simplex lies in global attraction of nonzero points onto the manifold.

In [19] Hirsch provided a streamlined set of conditions for the existence of the carrying simplex for continuous competitive maps, and later Ruiz-Herrera [30] proved the existence of the carrying simplex for maps under Hirsch's conditions. Ruiz-Herrera also provided conditions on the derivative of the map when it was continuously differentiable. To date, to the best of the author's knowledge, RuizHerrera's existence results for the carrying simplex remain the most general, and are based around showing that the boundary of repulsion of the origin attracts all nonzero orbits.

Here we obtain the existence of the carrying simplex for continuously differentiable maps by studying the evolution of hypersurfaces under repeated application of the map. A similar approach was used to study the geometry of carrying simplices for Lotka-Volterra differential equations $[6,4]$. The existence results that we obtain are essentially the same as Ruiz-Herrera in the case that $F$ is $C^{1}$ (Ruiz-Herrera relaxes this to $C^{0}$ ), but obtained via different means [30]. The approach used here is more aligned to that used to establish the existence of stable and unstable manifolds of maps, in that it uses the graph transform on spaces of Lipschitz functions, and invariance of cones (see, for example, [25]). We obtain global attraction of nonzero orbits to the carrying simplex by providing a measure of phase space volume that decreases under application of the map. 


\section{Existence of a carrying simplex}

We will show

Theorem 1. Let $F: C_{+} \rightarrow C_{+}$be defined by $F=D[I d] f$ where $f: C_{+} \rightarrow C_{+}$satisfies

AS1 $f$ is continuously differentiable on an open neighbourhood of $\mathrm{C}_{+}$;

$A S 2$ there are (precisely) $d$ axial points $q_{i} e_{i}$ for some $q_{i}>0, i \in I_{d}$;

AS3 $f(x) \gg 0, D f(x) \ll 0$ for $x \in C_{+}$;

AS4 the $d \times d$ matrix $M(x)=\left(\left(-\frac{x_{i}}{f_{i}(x)} \frac{\partial f_{i}(x)}{\partial x_{j}}\right)\right)$ has spectral radius $\rho(M(x))<1$ for all $x \in \Lambda:=[0, q]$;

Then the map $F$ has a carrying simplex.

(See Theorem 2 of Hirsch [19] and Corollary 6.1 with Remark 6.4 of Ruiz-Herrera [30]).

Remark 4. As mentioned already Ruiz-Herrera has shown this result as a corollary of a stronger result when $f$ is merely continuous [30]. His method does not use sequences of hypersurfaces, but rather shows directly from properties of competiitive maps that the boundary of the basin of repulsion of the origin is unordered, is asymptotically complete on $C_{+} \backslash\{0\}$ (and so attracts all points except the origin) and so is the carrying simplex. As it stands our method merely shows that the carrying simplex is globally attracting on $C_{+} \backslash\{0\}$. It would be interesting to see if asymptotic completeness of $\Sigma$ can be proved in our framework.

Remark 5. The assumptions AS2 and AS3 imply that the origin is an unstable node, and that for each $i \in I_{d}$ the axial fixed point $q_{i} e_{i}$ is globally asymptotically stable on the ray $(0, \infty) e_{i}$.

Remark 6. Due to AS3 $F$ cannot map a point $x \in \operatorname{int} C_{+}$to a point $F(x) \in \partial C_{+}$.

\subsection{Mappings of manifolds by normally monotone maps}

Let $\mathscr{U}$ denote the set of bounded $C^{1}$ hypersurfaces $S \subset \mathbb{R}_{+}^{d}$ with positive unit normals that project radially $1-1$ onto the unit probability simplex $\Delta_{d-1}$ and $\widehat{\mathscr{U}}$ the set of bounded $C^{1}$ hypersurfaces $S \subset \mathbb{R}_{+}^{d}$ with (nonzero) nonnegative unit normals that project radially $1-1$ onto $\Delta_{d-1}$. For each hypersurface $S \in \mathscr{U} \cup \widehat{\mathscr{U}}$ there is a radial function $R: \Delta_{d-1} \rightarrow \mathbb{R}, C^{1}$ on int $\Delta_{d-1}$, such that $\left\{S=\left\{R(u) u: u \in \Delta_{d-1}\right\}\right.$. Also set $\mathscr{U}_{0}=\left\{S \in \mathscr{U}: q_{i} e_{i} \in S, i \in I_{d}\right\}$, and similarly $\widehat{\mathscr{U}_{0}}=\left\{S \in \widehat{\mathscr{U}}: q_{i} e_{i} \in S, i \in I_{d}\right\}$. For $S \in \widehat{\mathscr{U}}$ we set $S_{-}=[0,1] S=\{\lambda x: \lambda \in[0,1], x \in S\}$. For $S \in \widehat{\mathscr{U}_{0}}$ the set $S_{-}$is a monotone region.

Let $\left\{v_{i}\right\}_{i=1}^{d-1} \subset T_{x} S$ (the tangent space to $S$ at $x$ ) be $d-1$ linearly independent vectors (i.e. $S$ is regular at $x$ ). The wedge product $n=\bigwedge\left(v_{1}, \ldots, v_{d-1}\right)$ is taken to be an outward normal to $S$ at $x$ where 


$$
\bigwedge\left(v_{1}, \ldots, v_{d-1}\right):=\operatorname{det}\left(\begin{array}{ccc}
v_{1}^{1} & \cdots & v_{1}^{d} \\
\vdots & \ddots & \vdots \\
v_{d-1}^{1} & \cdots & v_{d-1} \\
e_{1} & \cdots & e_{d}
\end{array}\right) \in \operatorname{span}\left\{e_{1}, \ldots, e_{d-1}\right\}
$$

The next lemma determines how such a unit normal of a $C^{1}$ hypersurface $S$ changes under the map $F$ at points where $D F$ is invertible.

Lemma 1. If $n$ is the unit outward normal to $S$ at $x$, and $D F$ is invertible at $x$ then the unit normal $n^{\prime}$ to $F(S)$ at $F(x)$ is given by $\frac{(D F(x))^{-T} n}{\left\|(D F(x))^{-T} n\right\|_{2}}$.

Proof. A tangent vector $v \in T_{x} S$ is mapped by $F$ to a tangent vector $D F(x) v \in$ $T_{F(x)} F(S)$. Then $n^{\prime} \cdot D F(x) v=\left(D F(x)^{T} n^{\prime}\right) \cdot v=0$ for all $v \in T_{x} S$. Hence $D F(x)^{T} n^{\prime}=$ $\mu n$ for some nonzero $\mu \in \mathbb{R}$ and $n^{\prime}=\mu D F(x)^{-T} n$ and the result follows by normalisation to a unit vector.

The following lemma shows that when $F$ is normally monotone it maps hypersurfaces with nonnegative normals to hypersurfaces with nonnegative normals (see lemma 4.1 [8]). This is just a restatement of the fact that normally monotone maps map the boundaries of normally monotone regions into boundaries of normally monotone regions.

Lemma 2. If $S \in \widehat{\mathscr{U}_{0}}$ and $(D F)^{-1}>0$ on $\Lambda$ then $F(S) \in \widehat{\mathscr{U}_{0}}$. If $S \in \widehat{\mathscr{U}_{0}}$ and $(D F)^{-1} \gg 0$ on $\Lambda \backslash \partial C_{+}$then $F(S) \in \mathscr{U}_{0}$.

Proof. By lemma 1 we only need to show that $F(S)$ projects onto $\Delta_{d-1}$. There exists a continuous function $R: \Delta_{d-1} \rightarrow \mathbb{R}_{+}$such that $S=\left\{R(u) u: u \in \Delta_{d-1}\right\}$. Then $F(S)=\left\{F(R(u) u): u \in \Delta_{d-1}\right\}$. Set $V_{i}(u)=f_{i}(R(u) u)$. We must show that for $v \in \Delta_{d-1}$ there exists a $u^{*} \in \Delta_{d-1}$ such that $u_{i}^{*} V_{i}\left(u^{*}\right)=v_{i} \bar{V}\left(u^{*}\right)$ for each $i \in I_{d}$ where $\bar{V}(u)=\sum_{k=1}^{d} u_{k} f_{k}(R(u) u)$.

We now use a differential equation with vector field $\xi$ to generate a flow which has a fixed point $u^{*}$. Consider the vector field $\xi: \Delta_{d-1} \rightarrow \mathbb{R}^{d}$ where $\xi_{i}(u):=$ $v_{i} \bar{V}(u)-u_{i} V_{i}(u)$ for $i \in I_{d}$. Then $\frac{d}{d t} \sum_{i=1}^{d} u_{i}=\sum_{i=1}^{d} \dot{u}_{i}=\sum_{i=1}^{d} \xi_{i}=0$, so that if $u(0) \in \Delta_{d-1}, \sum_{i=1}^{d} u_{i}(t)=1$ on the maximal forward time of solution existence. By using the variation of constants formula, we see that on the maximal time of solution existence $u_{k}(t) \geq 0$ whenever $u_{k}(0) \geq 0$, which combined with $\sum_{i=1}^{d} u_{i}=1$ gives global time existence and $u \in \Delta_{d-1}$ for all $t \geq 0$. The continuous flow defined by vector field $\xi$ maps $\Delta_{d-1}$ diffeomorphically into itself. Since $\Delta_{d-1}$ is compact, the flow has a fixed point $u^{*}$ (e.g. [2], theorem 3.8) where the vector field $\xi$ vanishes. By construction $\xi_{i}\left(u^{*}\right)=v_{i} \bar{V}\left(u^{*}\right)-u_{i}^{*} V_{i}\left(u^{*}\right)=0$. The second assertion follows since $\frac{(D F(x))^{-T} n}{\left\|(D F(x))^{-T} n\right\|_{2}} \gg 0$ when $n>0$.

Corollary 1. If $(D F)^{-1}>0$ on $\Lambda$ and $S \in \widehat{\mathscr{U}_{0}}$, then $F^{k}(S) \in \widehat{\mathscr{U}_{0}}$ for $k \in \mathbb{Z}_{+}$. If $(D F)^{-1} \gg 0$ on $\Lambda \backslash \partial C_{+}$and $S \in \widehat{\mathscr{U}_{0}}$, then $F^{k}(S) \in \mathscr{U}_{0}$ for $k \in \mathbb{Z}_{+}$. 
In the sequel we use $\pi_{k}: \mathbb{R}^{d} \rightarrow \mathbb{R}^{d-1}$ to denote the projection defined by $\pi_{k}(x)=$ $\left(x_{1}, \ldots, x_{k-1}, x_{k+1}, \ldots, x_{d}\right)$. Recall that

Definition 10 (Proper map). A map $g: \mathbb{R}^{d} \rightarrow \mathbb{R}^{d}$ is proper if whenever $A \subset \mathbb{R}^{d}$ is compact, $g^{-1}(A)$ is compact.

For example, a continuous map on a compact subset of $\mathbb{R}^{d}$ is proper. When properness of $g$ is combined with local invertibility and connectedness we obtain the important (e.g. chapter 3, section 1 in [1])

Theorem 2. Let $g: X \rightarrow Y$ be a proper and locally invertible map between metric spaces $X, Y$. Then for each $y \in Y$, the cardinality of the set $g^{-1}(y)$ is finite and constant on each connected component of $Y$.

Remark 7. Theorem 2 is a stepping stone to global inversion theorems on metric spaces. For example, when $g: X \rightarrow Y$ is a proper and locally invertible map between metric spaces $X, Y$, and in addition $X$ is arcwise connected and $Y$ simply connected, then $g$ is actually a homeomorphism from $X$ onto $Y$.

We can now apply Theorem 2 to establish that $S$ can be written as the graph of a function:

Lemma 3. If $S \in \mathscr{U}_{0}$ then $S$ can be written as the graph of a function hover $\pi_{d}(S)$.

Proof. The projection $\pi_{d}: S \rightarrow \pi_{d}(S) \subset \mathbb{R}_{+}^{d-1}$ is locally injective as the unit normal to $S$ is positive. Since $S$ is connected, $\pi_{d}(S)$ is connected. Moreover $\pi_{d}^{-1}(0)=\left\{q_{d}\right\}$ and hence $\pi_{d}: S \rightarrow \pi_{d}(S)$ is 1-1 from Theorem 2. This shows that $S$ is the graph of the $C^{1}$ function $h: \pi_{d}(S) \rightarrow \mathbb{R}$, where $h=\pi_{d}^{-1}$.

Lemma 4. If $S \in \mathscr{U}$ then $S$ is a the graph of a Lipschitz function with Lipschitz constant at most $\sqrt{d}$.

Proof. For $i=1, \ldots, d-1$ let $v^{i}$ be the row vector with $\left(v^{i}\right)_{j}=+1$ if $j=i$, $\left(v^{i}\right)_{j}=-1$ if $j=i+1$ and $\left(v^{i}\right)_{j}=0$ otherwise. Then $\left\{v_{i}\right\}_{i=1}^{d-1}$ spans $H$, where $H$ is the hyperplane with outward unit normal $\hat{e}=\frac{1}{\sqrt{d}}(1, \ldots, 1)$ that passes through the origin. From lemma 3, via a rotation, we may introduce coordinates $X_{1}, \ldots, X_{d-1}$ in $H$ so that each $x \in S$ can be written as $x=\sum_{i=1}^{d-1} v^{i} X_{i}+\phi\left(X_{1}, \ldots, X_{d-1}\right) \hat{e}$, for some $C^{1}$ real-valued function $\phi: \mathbb{R}^{d-1} \rightarrow \mathbb{R}$. Then $\partial x / \partial X_{i}=v^{i}+\left(\partial \phi / \partial X_{i}\right) \hat{e}$ and $q=\partial x / \partial X_{1} \wedge \cdots \wedge \partial x / \partial X_{d-1} \gg 0$, since the product is normal to the hypersurface. This yields

$$
\begin{aligned}
q & =\left(v^{1}+\phi_{X_{1}} \hat{e}\right) \wedge\left(v^{2}+\phi_{X_{2}} \hat{e}\right) \wedge \cdots \wedge\left(v^{d-1}+\phi_{X_{d-1}} \hat{e}\right) \\
& =\left(\bigwedge_{i=1}^{d-1} v^{i}\right)+\phi_{X_{1}} \hat{e} \wedge v^{2} \wedge \cdots \wedge v^{d-1}+\cdots+\phi_{X_{d-1}} v^{1} \wedge v^{2} \wedge \cdots \wedge v^{d-2} \wedge \hat{e}
\end{aligned}
$$

It is straightforward to compute $\bigwedge_{i=1}^{d-1} v^{i}=e$ and we have, for $j=1, \ldots, d-1$, 


$$
\begin{aligned}
q_{j}-q_{j+1} & =\phi_{X_{j}} v^{j} \cdot\left(v^{1} \wedge \cdots \wedge v^{j-1} \wedge \hat{e} \wedge v^{j+1} \wedge \cdots \wedge v^{d-1}\right) \\
& =-\phi_{X_{j}} \hat{e} \cdot\left(v^{1} \wedge \cdots \wedge v^{j-1} \wedge v^{j} \wedge v^{j+1} \wedge \cdots \wedge v^{d-1}\right) \\
& =-\sqrt{d} \phi_{X_{j}} .
\end{aligned}
$$

But $q_{i}>0$ for each $i \in I_{d}$ and $\sum_{i=1}^{d} q_{i}=d$ (established by taking the dot product of (5) with $e$ ) so that each $q_{i} \in(0, d)$. Thus for $j \in I_{d-1}$ we may bound $\phi_{X_{j}}=-\frac{1}{\sqrt{d}}\left(q_{j}-\right.$ $\left.q_{j+1}\right) \in(-1,1)$. This shows that $\phi$ is Lipschitz with Lipschitz constant at most $\sqrt{d}$.

Set $\mathscr{K}_{0}=\left\{T \in \mathscr{K}: T_{i}\left(q_{i} e_{i}\right)=q_{i} e_{i}, i \in I_{d}\right\}$. Let $\pi_{H}(x)$ denote the projection of $x \in \mathbb{R}^{d}$ onto $H$ along $\hat{\varepsilon}$. Set $Q=\pi_{H}(\Lambda)$ and define the set $\mathscr{L}_{0}$ of functions $\phi: Q \rightarrow \mathbb{R}$ which are Lipschitz with Lipschitz constant at most $\sqrt{d}$ and such that $\phi\left(q_{i} e_{i}\right)=\sqrt{d} q_{i}$ for $i \in I_{d} . \mathscr{L}_{0}$ is compact in the topology of uniform convergence.

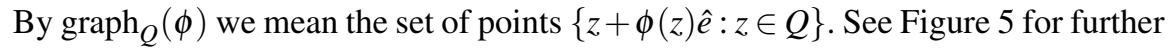
explanation.

Lemma 5. For each $S \in \mathscr{U}_{0}$ there is a $\phi \in \mathscr{L}_{0}$ such that $S=\operatorname{graph}_{Q}(\phi) \cap C_{+}$.

Proof. Use the construction of lemma 4.
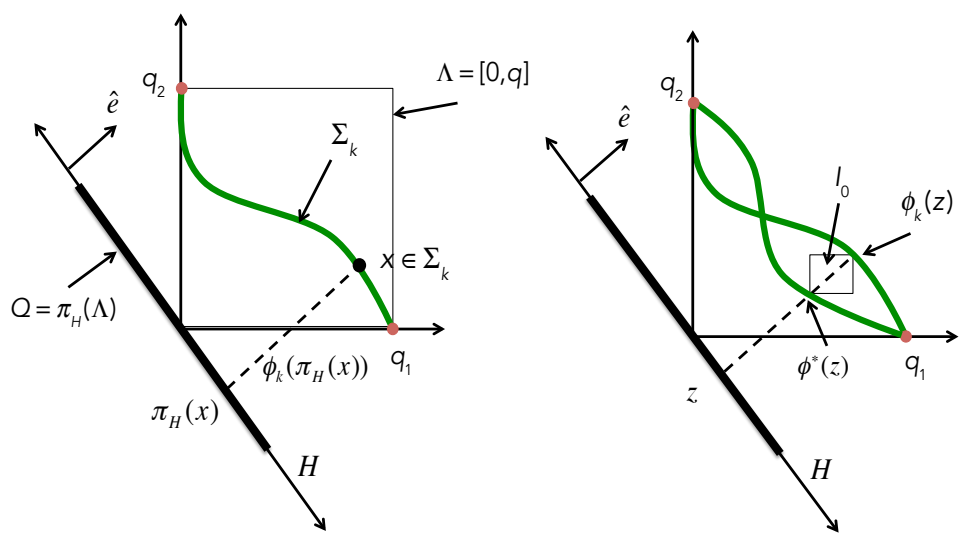

Fig. 5 The various coordinates used to describe the manifolds $\Sigma_{k}=\operatorname{graph}_{Q} \phi_{k}$ in the proof of Theorem 1 .

Next we show that $\Lambda$ is a forward invariant set of $F$. 
Lemma 6. Under assumptions AS2, AS3, and AS4, $F(\Lambda) \subset \Lambda$. Moreover, boundary points of $\Lambda$ that are not axial fixed points are mapped inside $\Lambda$ by $F$.

Proof. We must show that $F(x) \leq q$ whenever $x \leq q$. Write $x=s \star q:=\left(s_{1} q_{1}, \ldots, s_{d} q_{d}\right)$ where $s \in[0, e]$. Then $F_{1}(x)-q_{1}=q_{1}\left(s_{1} f_{1}(s \star q)-1\right)$. Notice that since $D f \ll 0$, $s_{1} f_{1}(s \star q)-1<s_{1} f_{1}\left(s_{1} q_{1}, 0, \ldots, 0\right)-1$. Now set $\theta_{1}(t)=t f_{1}\left(t q_{1}, 0, \ldots, 0\right)-1$ so that $\theta(1)=0$ by AS2. Then $\left.\theta_{1}^{\prime}(t)=f_{1}\left(t q_{1}, 0, \ldots, 0\right)+t q_{1} \frac{\partial f_{1}}{\partial x_{j}}\left(t q_{1}, 0, \ldots, 0\right)\right)=$ $f_{1}\left(t q_{1}, 0, \ldots, 0\right)\left(1-M_{11}\left(t q_{1}, 0, \ldots, 0\right)\right)$. We know by AS4 that $\rho\left(M\left(t q_{1}, 0, \ldots, 0\right)\right)<$ 1 and hence $M_{11}\left(t q_{1}, 0, \ldots, 0\right)<1$. This show that $\theta_{1}^{\prime}(t)>0$ for $t \in[0,1]$ and so using $\theta_{1}(1)=0, \theta_{1}(t)<0$ for $t \in[0,1)$. Hence $F_{1}(x)-q_{1}=q_{1}\left(s_{1} f_{1}(s \star q)-1\right)<0$ for all $s \in[0, e]$ with $s_{1}<1$. Similarly for the other $F_{j}(x)-q_{j}$ for $j=2, \ldots, d$.

For the second part, suppose that $x \in \partial \Lambda \backslash \cup_{j=1}^{d}\left\{q_{j} e_{j}\right\}$. Then $x=s \star q$ where there is a subset $J \subseteq I_{d}$ for which $s_{j}=1$ for $j \in J$, and $s \neq e_{j}$ for any $j \in I_{d}$. For $j \in J$ we have $F_{j}(x)-q_{j}=q_{j}\left(f_{j}(s \star q)-1\right)$. Since $s \neq e_{j}$, we obtain $q_{j}\left(f_{j}(s \star q)-1\right)<$ $q_{j}\left(f_{j}\left(q_{j} e_{j}\right)-1\right)=0$. For $j \notin J$ we have $F_{j}(x)-q_{j}=q_{j}\left(s_{j} f_{j}(s \star q)-1\right)<q_{j}\left(f_{j}(s \star\right.$ $q)-1)<0$. Hence $F(x) \in C_{+} \backslash \Lambda$.

Lemma 6 enables us to first consider dynamics restricted to $\Lambda$. Later we show that all points in $C_{+}$eventually enter and stay in $\Lambda$.

\section{AS3 and AS4 provide for a decreasing phase space measure}

Define the measure $\mu$ via $\mu(P)=\int_{P} \prod_{i=1}^{d} x_{i}^{-1} \mathrm{~d} x$ for each Lebesgue measurable set $P \subseteq \Lambda$. Note that the integral may not be finite if $P$ intersects the boundary $\partial C_{+}$. However, the following lemma shows that the $\mu$-measure of the volume between two bounded hypersurfaces $S_{1}, S_{2} \in \mathscr{U}$ is bounded. We may represent $S_{i}=\left\{R_{i}(u) u\right.$ : $\left.u \in \Delta_{d-1}\right\}$ for bounded $R_{i}, i=1,2$. Since the normal is positive for hypersurfaces in $\mathscr{U}$, the gradient of each $R_{i}$ is bounded.

Lemma 7. Let $R_{i}: \Delta_{d-1} \rightarrow \mathbb{R}, i=1,2$ be positive and bounded $C^{1}$ functions such that $R_{1}(u)=R_{2}(u)$ for all $u \in \partial \Delta_{d-1}$. Let $\Theta=\left\{(u, R) \in \Delta_{d-1} \times \mathbb{R}_{+}: \min \left\{R_{1}(u), R_{2}(u)\right\} \leq\right.$ $\left.R \leq \max \left\{R_{1}(u), R_{2}(u)\right\}\right\}$ and $v$ denote the volume element on $\Delta_{d-1}$. Then

$$
\int_{\Theta} d \mu=\int_{\Delta_{d-1}} \frac{1}{\prod_{i=1}^{d} u_{i}}\left|\log R_{1}(u)-\log R_{2}(u)\right| d v(u)
$$

is finite.

Proof.

$$
\begin{aligned}
\int_{\Theta} \mathrm{d} \mu & =\int_{\Theta} \frac{1}{\prod_{i=1}^{d} x_{i}} \mathrm{~d} x \\
& =\int_{\Delta_{d-1}} \int_{R_{1}(u)}^{R_{2}(u)} \frac{1}{R \prod_{i=1}^{d} u_{i}} \operatorname{sgn}\left(R_{1}(u)-R_{2}(u)\right) \mathrm{d} R \mathrm{~d} v(u) \\
& =\int_{\Delta_{d-1}} \frac{1}{\prod_{i=1}^{d} u_{i}}\left|\log R_{1}(u)-\log R_{2}(u)\right| \mathrm{d} v(u)
\end{aligned}
$$


Since $\Delta_{d-1}$ is compact, the only problem points are on the boundary $\partial \Delta_{d-1}$ where $\prod_{i=1}^{d} u_{i}$ vanishes. However, $\left|\log R_{1}(u)-\log R_{2}(u)\right|$ also vanishes on $\partial \Delta_{d-1}$, so that $\left|\log R_{1}(u)-\log R_{2}(u)\right|=\left(\prod_{i=1}^{d} u_{i}^{\beta_{i}}\right) W(u)$ for some set of $\beta_{i}>0$ and some bounded, nonnegative and continuous function $W: \Delta_{d-1} \rightarrow \mathbb{R}$. Hence

$$
\begin{aligned}
\int_{\Theta} \mathrm{d} \mu & =\int_{\Delta_{d-1}} \prod_{i=1}^{d} u_{i}^{\beta_{i}-1}|W(u)| \mathrm{d} v(u) \\
& \leq \max _{u \in \Delta_{d-1}}|W(u)| \int_{\Delta_{d-1}} \prod_{i=1}^{d} u_{i}^{\beta_{i}-1} \mathrm{~d} v(u) \\
& =\max _{u \in \Delta_{d-1}}|W(u)|\left(\frac{\prod_{i=1}^{d} \operatorname{Gamma}\left(\beta_{i}\right)}{\operatorname{Gamma}\left(\sum_{i=1}^{d} \beta_{i}\right)}\right)
\end{aligned}
$$

which is finite.

Lemma 8. Under the assumption of AS4

$$
0<\operatorname{det}\left(I+D[x] D[f(x)]^{-1} D f(x)\right)<1 \quad x \in \Lambda \backslash\{0\} .
$$

Proof. Let $M=-D[x] D[f(x)]^{-1} D f(x) \gg 0$ and recall that $\rho(M)<1$. Consider $A=$ $I-M$. Then $A$ is an $M$-matrix (see section 4, definition 11). Moreover, $(1-\varepsilon) I \geq A$ for sufficiently small $\varepsilon>0$ and so using Theorem 2.5.4 of [20], $0<\operatorname{det} A \leq \operatorname{det}((1-$ $\varepsilon) I)<1$, i.e. $0<\operatorname{det}(I-M)<1$.

Now we consider how the $\mu$-measure of a subset of $C_{+}$decreases with each application of $F$.

Lemma 9. Under the assumption AS4, the $\mu$-measure of a measurable set $P \subseteq \Lambda$ of finite $\mu$-measure strictly decreases under $F$.

Proof. We compute

$$
\begin{aligned}
\int_{F(P)} \mathrm{d} \mu & =\int_{P} \prod_{i=1}^{d}\left(F_{i}(x)\right)^{-1} \operatorname{det} D F(x) \mathrm{d} x \\
& =\int_{P} \prod_{i=1}^{d}\left(x_{i} f_{i}(x)\right)^{-1} \operatorname{det}(D[f(x)]) \operatorname{det}\left(I+D[x] D[f(x)]^{-1} D f(x)\right) \mathrm{d} x \\
& =\int_{P} \prod_{i=1}^{d} x_{i}^{-1} \operatorname{det}\left(I+D[x] D[f(x)]^{-1} D f(x)\right) \\
& \leq \kappa \int_{P} \prod_{i=1}^{d} x_{i}^{-1} \mathrm{~d} x \\
& <\int_{P} \mathrm{~d} \mu
\end{aligned}
$$

where inequality $A$ follows from lemma 8. 
Given a function $f: Q \rightarrow \mathbb{R}$, by $\operatorname{epi}_{Q} f$ we mean the set $\operatorname{epi}_{Q} f=\{(z, r): z \in Q, r \geq$ $f(z)\}$. Also for two sets $A, B, A \triangle B=(A \backslash B) \cup(B \backslash A)$ denotes their symmetric difference.

Corollary 2. Let $S_{0}, T_{0} \in \mathscr{U}_{0}$ and $S_{k}=F^{k}\left(S_{0}\right), T_{k}=F^{k}\left(T_{0}\right)$. Suppose $S_{k}=$ graph $_{Q} \phi_{k}$ and $T_{k}=\operatorname{graph}_{Q} \psi_{k}$. Let $\Theta_{k}=e p i_{Q} \phi_{k} \triangle e p i_{Q} \psi_{k}$. Then $\mu\left(\Theta_{k}\right) \rightarrow 0$ as $k \rightarrow \infty$.

\subsection{Proof of existence of an invariant hypersurface $\Sigma \subset \Lambda$}

Lemma 10. Under the assumptions AS1 - AS4 there exists an invariant hypersurface $\Sigma \in \widehat{\mathscr{U}}_{0}$ and $\Sigma$ attracts $\Lambda \backslash\{0\}$.

Proof. Let $\Sigma_{0} \in \mathscr{U}_{0}$ and suppose that $\Sigma_{0}=\operatorname{graph}_{Q} \phi_{0}$. Then $\Sigma_{k} \in \mathscr{U}_{0}$ where $\Sigma_{k}=$ $F^{k}\left(\Sigma_{0}\right)$ and there is a sequence $\left\{\phi_{k}\right\}_{k=0}^{\infty}$ such that $\Sigma_{k}=\operatorname{graph}_{Q} \phi_{k}$. By compactness of $\mathscr{L}_{0}$ there exists a $\phi^{*} \in \mathscr{L}_{0}$ and a subsequence $\phi_{t_{k}} \rightarrow \phi^{*}$ as $k \rightarrow \infty$ (uniformly). Set $\Sigma=\operatorname{graph}_{Q}\left(\phi^{*}\right) \cap C_{+} \in \widehat{\mathscr{U}_{0}}$. We will show that $\phi_{k} \rightarrow \phi^{*}$ uniformly as $k \rightarrow \infty$. Using $\|\cdot\|_{0}$ to denote the supremum norm, by the triangle inequality

$$
\left\|\phi_{k}-\phi^{*}\right\|_{0} \leq\left\|\phi_{k}-\phi_{t_{k}}\right\|_{0}+\left\|\phi_{t_{k}}-\phi^{*}\right\|_{0}
$$

and we already know that $\left\|\phi_{t_{k}}-\phi^{*}\right\|_{0} \rightarrow 0$ as $k \rightarrow \infty$. On the other hand, we will now show that since by corollary $2, \mu\left(\operatorname{epi}_{Q} \phi_{k} \triangle \mathrm{epi}_{Q} \phi_{t_{k}}\right) \rightarrow 0$, we must also have $\| \phi_{k}-$ $\phi_{t_{k}} \|_{0} \rightarrow 0$ as $k \rightarrow \infty$. For suppose that $\mu\left(\operatorname{epi}_{Q} \phi_{k} \triangle \mathrm{epi}_{Q} \phi_{t_{k}}\right) \rightarrow 0$, but that there exists a point $z \in Q$ such that $\left|\phi_{k}(z)-\phi_{t_{k}}(z)\right| \not \rightarrow 0$ as $k \rightarrow \infty$. Then there exists an $\eta>0$, an integer $N(z)$, and a subsequence $k_{j} \rightarrow \infty$ as $j \rightarrow \infty$ such that $\left|\phi_{k_{j}}(z)-\phi_{t_{k_{j}}}(z)\right| \geq \eta>0$ for all $j \geq N(z)$. Since each graph $\phi_{\phi_{j}}$ and $\operatorname{graph}_{Q} \phi_{t_{k_{j}}}$ are are hypersurfaces in $\mathscr{U}_{0}$ (so that they decrease in each coordinate direction) for each $j \geq N(z)$ there exists a rectangle $I_{0} \subset \operatorname{epi}_{Q} \phi_{k_{j}} \triangle \mathrm{epi}_{Q} \phi_{t_{k_{j}}}$ of positive $\mu$-measure (see Figure 5). But this contradicts that $\mu\left(\mathrm{epi}_{Q} \phi_{k_{j}} \triangle \mathrm{epi}_{Q} \phi_{t_{k_{j}}}\right) \rightarrow 0$ as $j \rightarrow \infty$, showing by contradiction that there is pointwise convergence of $\left\{\phi_{k}\right\}_{k=0}^{\infty}$. Since each $\phi_{k}$ and $\phi^{*}$ are Lipschitz we actually have $\phi_{k} \rightarrow \phi^{*}$ uniformly as $k \rightarrow \infty$. By construction $\Sigma=\operatorname{graph} \phi^{*}$ is in $\mathscr{U}_{0}$ and is invariant.

Now suppose that $x \in \Lambda \backslash \partial \Lambda \backslash\{0\}$. By induction on the dimension $d$, if $x \in \partial C_{+}$, then $x$ is attracted to $\partial \Sigma$ (Theorem 1 is well-known to be true for $d=1$ ). Otherwise there exists a $\Sigma_{0} \in \mathscr{U}_{0}$ such that $x \in \Sigma_{0}$ and since $F^{k}\left(\Sigma_{0}\right)$ converges uniformly to $\Sigma$ as $k \rightarrow \infty, x$ is attracted to $\Sigma$.

To this point we have shown that for any $\Sigma_{0} \in \mathscr{U}_{0}$, the sequence $\left\{F^{k}\left(\Sigma_{0}\right)\right\}_{k=0}^{\infty}$ converges uniformly to an invariant and hypersurface $\Sigma \in \mathscr{U}_{0}$ that is the graph of a Lipschitz function with Lipschitz constant at most $\sqrt{d}$. Now we must show that all points outside $\Lambda$ eventually enter and remain in $\Lambda$, and also to identify $\Sigma$ with the carrying simplex we also need to show that $\Sigma$ is unordered and asymptotically complete. 
We have the following for $F=D[\mathrm{Id}] f$ : (e.g., see [30], lemma 6.1.)

Lemma 11. Under the assumptions AS3 and AS4, for $x \in C_{+}, \omega(x) \subset \Lambda$.

Proof. Choose $x \in C_{+} \backslash\{0\}$ and consider the orbit $O^{+}(x)$. Since $O^{+}(x)$ is bounded, its closure is compact and $\omega(x)$ is nonempty. For $x_{1}>0, x \geq\left(x_{1}, 0, \ldots, 0\right)$ and so $f_{1}(x) \leq f_{1}\left(x_{1}, 0, \ldots, 0\right)$. Hence $F(x)_{1}=x_{1} f_{1}(x) \leq x_{1} f_{1}\left(x_{1}, 0, \ldots, 0\right)$ and $F^{k+1}(x)_{1} \leq$ $F^{k}(x)_{1} f_{1}\left(F^{k}(x)_{1}, 0, \ldots, 0\right)$. Thus if $y^{k}:=F^{k}(x)$ and $G_{j}: \mathbb{R}_{+} \rightarrow \mathbb{R}_{+}$is given by $G_{j}(s)=s f_{j}\left(s e_{j}\right), y_{1}^{k+1} \leq G_{1}\left(y_{1}^{k}\right)$ for $k \in \mathbb{Z}_{+}$and similarly we find $0 \leq y_{j}^{k+1} \leq G_{j}\left(y_{j}^{k}\right)$ for $k \in \mathbb{Z}_{+}$, for $j=2, \ldots, d$.

Let us focus on the scalar difference inequality

$$
0 \leq p^{k+1} \leq G_{1}\left(p^{k}\right)
$$

with $p^{0} \in \mathbb{R}_{+}$given. Hence $p^{k+1}-q_{1} \leq G_{1}\left(p^{k}\right)-q_{1}$ from which it follows that if $p^{0} \leq q_{1}$ then $p^{k} \leq q_{1}$ for all $k \in \mathbb{Z}_{+}$. On the other hand, if $p^{0}>q_{1}$ then either $p^{n}<q_{1}$ for some $n \in \mathbb{Z}_{+}$with $n \geq 1$, in which case $p^{k}<q_{1}$ for all $k \geq n$, or $p^{k} \geq q_{1}$ for all $k \in \mathbb{Z}_{+}$. In the latter case $q_{1} \leq p^{k+1} \leq G_{1}\left(p^{k}\right)<p^{k}$ for $p^{k}>q_{1}$, since from AS4 we find that $s<G_{j}(s)<q_{j}$ for all $s \in\left(0, q_{j}\right), G_{j}\left(q_{j}\right)=q_{j}$ and $G_{j}(s)<s$ for $s>q_{j}$, and $p^{k} \rightarrow q_{1}$. Hence we see that for any $\theta \in \omega(x)$ we have $\theta_{i} \leq q_{i}$ for $i=1, \ldots, d$, i.e. $\omega(x) \subset \Lambda$.

Lemma 12. If $S \in \mathscr{U}$ then $S$ is unordered.

Proof. Write $S=\operatorname{graph} h$ where $h$ is $C^{1}$. The unit normal to $S$ at $x$ is $n(x)=$ $\left(-D h\left(\pi_{d}(x)\right), 1\right) /\left\|\left(-D h\left(\pi_{d}(x)\right), 1\right)\right\|$. Since the unit normal is positive, $D h \ll 0$ on $\pi_{d}(S)$. Now we show that when $x, y \in S$ with $\left(\pi_{d}(x)\right)_{i}>\left(\pi_{d}(y)\right)_{i}$ for $i \in I_{d-1}$ then $t \pi_{d}(x)+(1-t) \pi_{d}(y) \in \pi_{d}(S)$ for all $t \in[0,1]$. Suppose not and let $t^{*} \in[0,1]$ be the largest value of $t \in[0,1]$ such that $t \pi_{d}(x)+(1-t) \pi_{d}(y) \in \partial\left(\pi_{d}(S)\right)$. Then $h\left(t^{*} \pi_{d}(x)+\left(1-t^{*}\right) \pi_{d}(y)\right)=0$ and $t^{*} \pi_{d}(x)+\left(1-t^{*}\right) \pi_{d}(y)<x$ which implies that $h(x)<0$ (since $D h \ll 0)$, contradicting that $(x, h(x)) \in C_{+}$.

For $x, y \in S$ distinct $x-y=\left(\pi_{d}(x)-\pi_{d}(y), h\left(\pi_{d}(x)\right)-h\left(\pi_{d}(y)\right)\right)$. If $x>y$ then $x_{i} \geq y_{i}$ for $i=1, \ldots, d-1$ and $h\left(\pi_{d}(x)\right) \geq h\left(\pi_{d}(y)\right)$. We know that $\pi_{d}$ is $1-1$, so we need only consider when $x_{i}>y_{i}$ for at least one $i=1, \ldots, d-1$ (and $x_{i}=y_{i}$ otherwise). But in that case $h$ (strictly) decreases along the line segment joining $\pi_{d}(x), \pi_{d}(y)$ thus contradicting that $h\left(\pi_{d}(x)\right)>h\left(\pi_{d}(y)\right)$. Hence $x, y$ are not ordered.

Lemma 13. The manifold $\Sigma$ is unordered.

Proof. $\Sigma$ is the graph of a locally Lipschitz function $h: \pi_{d}(\Sigma) \rightarrow \mathbb{R}$, and by lemma 12 , and taking limits, $h$ is nowhere increasing in each coordinate direction and there are no points in $\Sigma$ that are strongly ordered, i.e. there are no points $u, v \in \Sigma$ with $u \gg v$ or $v \gg u$. Suppose there is $x, y \in \Sigma$ with $x>y$. Then we need $x_{i} \geq y_{i}$ for $i=$ $1, \ldots, d-1$ (with at least one strict inequality) and $h\left(\pi_{d}(x)\right)=h\left(\pi_{d}(y)\right)$; by lemma 12 actually we need $h\left((1-\varepsilon) \pi_{d}(x)+\varepsilon \pi_{d}(y)\right)$ is constant for $\varepsilon \in[0,1]$ which implies that $x_{\varepsilon}:=(1-\varepsilon) x+\varepsilon y \in \Sigma$ for $\varepsilon \in[0,1]$. Then for $\varepsilon>0$ small enough $F$ is invertible on the open ball $B(x ; \varepsilon)$ and $F^{-1}(x)-F^{-1}\left(x_{\varepsilon}\right)=\int_{0}^{1} D F^{-1}\left(s x+(1-s) x_{\varepsilon}\right) \mathrm{d} s\left(x-x_{\varepsilon}\right)$. 
But $\int_{0}^{1} D F^{-1}\left(s x+(1-s) x_{\varepsilon}\right) \mathrm{d} s \gg 0$ and so $x_{\varepsilon}>x$ gives $F^{-1}\left(x_{\varepsilon}\right)-F^{-1}(x) \gg 0$. But since $\Sigma$ is invariant we then have two points $F^{-1}(x), F^{-1}\left(x_{\varepsilon}\right) \in \Sigma$ that are strongly ordered, a contradiction.

Finally we note that $\Sigma$ is asymptotically complete on $C_{+} \backslash\{0\}$, i.e. CS2 in the defintion of the carrying simplex: For every $x \in C_{+} \backslash\{0\}$ the trajectory of $x$ is asymptotic to the trajectory of some $y \in \Sigma: \lim _{n \rightarrow \infty}\left|F^{n}(x)-F^{n}(y)\right|=0$. This is a stronger condition than global attraction of orbits onto $\Sigma$. Rather it shows that for every orbit $\Gamma$ in $C_{+} \backslash \Sigma$ there is an orbit actually in $\Sigma$ that 'shadows' $\Gamma$. Note that we cannot simply project $\Gamma$ onto $\Sigma$ as the projected points will not correspond to an orbit in $\Sigma$. Asymptotic completeness is nicely shown in [30], and we simply outline the idea. First it is shown that for any two nonzero points $x, y \in C_{+}$, for which $F^{k}(x) \geq F^{k}(y)$ for all $k \in \mathbb{Z}_{+}$, we have $\left|F^{k}(x)-F^{k}(y)\right| \rightarrow 0$ as $k \rightarrow \infty$. This step is shown in lemma 6.5 [30]. Alternatively, one can show this by using that phase space $\mu$-measure decreases with $k$. Next, Ruiz-Herrera shows [30][page 16] that for any $x \in C_{+} \backslash \Sigma$ there does indeed exist an initial point $y \in \Sigma$ such that $F^{k}(x)>F^{k}(y)$ for all $k \in \mathbb{Z}_{+}$, thus establishing asymptotic completeness of $\Sigma$.

\section{Conclusion: Proof of Theorem 1}

1. By lemma $6, \Lambda$ is forward invariant under $F$;

2. By lemma 10 there is an invariant manifold $\Sigma \in \widehat{\mathscr{U}_{0}}$ that attracts points in $\Lambda \backslash\{0\}$ $0 \not n \Sigma$ as the origin is repelling;

3. By lemma 11 all points in $C_{+}$enter and stay in $\Lambda$ and moreover, by lemma 6 points on the relative boundary of $\Lambda$ that are not axial fixed points move interior to $\Lambda$ (so that such $F$-mapped points belong to a hypersurface $S \in \mathscr{U}_{0}$ ). Hence $\Sigma$ attracts $C_{+} \backslash\{0\}$

4. By lemma $13 \Sigma$ is unordered.

5. From the last paragraph $\Sigma$ is asymptotically complete.

\section{Some relationships between normally monotone and competitive maps}

Here we briefly discuss the connections between normally monotone maps and competitive maps using results for the the well-known class of $P$-matrices. We recall the following

Definition 11 ( $M$-matrix). A real square matrix $A$ is an $M$-matrix if it can be expressed in the form $A=s I-B$ where $B \geq 0$ with $s \geq \rho(B)$.

Definition 12 ( $P$-matrix). A real square matrix $A$ is a $P$-matrix if all its principal minors are positive.

The following standard result (see for example [9]) will be useful below.

Lemma 14. If $A$ is a nonsingular $M-$ matrix, it is also a $P$-matrix. 
Lemma 15. Under assumption $A S 3$, for each $x \in$ int $_{+},(D F)^{-1}(x) \gg 0$ if and only if $\rho(M(x))<1$ where $M(x)=-D[x / f(x)] D f(x)$.

Proof. $D F=D[f]+D[\operatorname{Id}] D f=D[f](I+D[\operatorname{Id}] D g)$ where $g=\log f=\left(\log f_{1}, \ldots, \log f_{d}\right)$. $(D F)^{-1}=(I+D[\operatorname{Id}] D g)^{-1} D[f]^{-1}$ and since $f \gg 0$ we have $(D F)^{-1} \gg 0 \Leftrightarrow$ $(I+D[\operatorname{Id}] D g)^{-1}=(I-M)^{-1} \gg 0$ where $M=-D[\operatorname{Id}] D g$. If $\rho(M)<1$, the inverse $(I-M)^{-1}$ exists and equals the convergent expansion $\sum_{k=0}^{\infty} M^{k}$, so that when $M \gg 0$, such as when $x \in \operatorname{int} \Lambda,(D F)^{-1} \gg 0$. By continuity, on $C_{+}$, when we only know that $M \geq 0$, it is certainly true that $(D F)^{-1}>0$ (but note when $M$ is irreducible, so that a positive power of $M$ is a positive matrix, $\left.(D F)^{-1} \gg 0\right)$.

Conversely if $(D F)^{-1} \gg 0$, by the Perron-Frobenius theorem for positive matrices, $M$ has a positive eigenvalue equal to its spectral radius $\rho(M)$ and a unique positive eigenvector $v$. Since we know that $D F$ is nonsingular, $\rho(M) \neq 1$. Then $(I-M)^{-1} v=(1-\rho(M))^{-1} v \gg 0$, since $(I-M)^{-1} \gg 0$ and $v \gg 0$, so we must have $\rho(M)<1$. If we only know that $(D F)^{-1}>0$, then we still need $\rho(M)<1$.

We let $\left.F\right|_{\Lambda}: \Lambda \rightarrow F(\Lambda)$ denote the restriction of $F$ to $\Lambda$.

Lemma 16. Under assumptions AS3, AS4

1. $\left.F\right|_{\Lambda}: \Lambda \rightarrow F(\Lambda)$ is a homeomorphism;

2. $\left.F\right|_{\Lambda}: \Lambda \rightarrow F(\Lambda)$ is competitive on $\Lambda$ and strongly competitive on $\Lambda \backslash \partial C_{+}$;

Proof.

1. 1 From AS3 and AS4, $D F(x)=D[f(x)]+D[x] D f(x)$ is an $M$-matrix (what Gale and Nikaidô call Leontief-type) and hence also a $P$-matrix for $x \in \Lambda$. Since $\Lambda$ is rectangular, the univalence theorem of Gale and Nikaidô [14] shows that $\left.F\right|_{\Lambda}$ is injective, and hence is a bijection $\Lambda \rightarrow F(\Lambda)$. Since $\Lambda$ is compact, $\left.F\right|_{\Lambda}$ is a homeomorphism of $\Lambda$ onto $F(\Lambda)$.

2. $D F$ is an $M-$ and $P$-matrix. From the previous part, $\left.F\right|_{\Lambda}$ has an inverse $\left.F\right|_{\Lambda} ^{-1}$. According to theorem 4.4 in [14] this implies that $\left.F\right|_{\Lambda} ^{-1}$ is monotone, i.e. that $F(x) \leq F(y)$ implies $x \leq y$ for $x, y \in \Lambda$, so that $\left.F\right|_{\Lambda} ^{-1}$ is competitive on $\Lambda$. However the proof of theorem 4.4 in [14] requires only $\partial F_{i} / \partial x_{j} \leq 0$ for $i \neq j$ and if this is strengthened to $\partial F_{i} / \partial x_{j}<0 i \neq j$, as is valid in our case when $x \in \Lambda \backslash \partial C_{+}$, and the proof followed through we then obtain $F(x)<F(y)$ implies $x \ll y$ for $x, y \in \Lambda \backslash \partial C_{+}$.

Remark 8. For a more general result regarding when competitive maps are global homeomorphisms, see [38].

\subsection{Some inequalities for the spectral radius}

The spectral radius of a matrix $M=\left(\left(m_{i j}\right)\right)$ is bounded by any matrix norm of that matrix: $\rho(M) \leq \max _{\|x\|=1}\|M x\|$ and so in particular $\rho(M) \leq\|M\|_{1}=$ $\max _{1 \leq j \leq d} \sum_{i=1}^{d}\left|m_{i j}\right|, \rho(M) \leq\|M\|_{\infty}=\max _{1 \leq i \leq d} \sum_{j=1}^{d}\left|m_{i j}\right|$, and $\rho(M) \leq\|M\|_{\max }=$ $\max _{1 \leq i, j \leq d}\left|m_{i j}\right|$. 
Lemma 17. With $M(x)=-D[x] D[F(x)]^{-1} D f(x)$, where $f \gg 0$ and $D f \ll 0$,

$$
\begin{aligned}
& \rho(M(x)) \leq\|M(x)\|_{1}=\max _{1 \leq j \leq d} \sum_{i=1}^{d}-\frac{x_{i}}{f_{i}(x)} \frac{\partial f_{i}}{\partial x_{j}}(x), \\
& \rho(M(x)) \leq\|M(x)\|_{\infty}=\max _{1 \leq i \leq d} \frac{x_{i}}{f_{i}(x)} \sum_{j=1}^{d}-\frac{\partial f_{i}}{\partial x_{j}}(x),
\end{aligned}
$$

and

$$
\rho(M(x)) \leq\|M(x)\|_{\max }=\max _{1 \leq i, j \leq d}-\frac{x_{i}}{f_{i}(x)} \frac{\partial f_{i}}{\partial x_{j}}(x),
$$

Lemma 18. For any invertible $d \times d$ diagonal matrix $D$, and any $d \times d$ matrix $P$, $\rho(P)<1$ if and only if $\rho\left(D^{-1} P D\right)<1$.

Proof. $\rho(P)<1 \Leftrightarrow \sum_{k=0}^{\infty} P^{k}$ converges $\Leftrightarrow D^{-1}\left(\sum_{k=0}^{\infty} P^{k}\right) D=\sum_{k=0}^{\infty}\left(D^{-1} P D\right)^{k}$ converges $\Leftrightarrow \rho\left(D^{-1} P D\right)<1$.

\section{More examples from Ecology}

A ready source of Kolmogorov maps is Ecology where such a map $F: C_{+} \rightarrow C_{+}$ models the change in population density of $d$ species in one generation (assuming that generations do not overlap). We mentioned two such examples in the introduction. Now we consider these same models in higher dimensions.

\subsection{The Leslie-Gower model}

Here the map takes the form $F=\left(F_{1}, \ldots, F_{d}\right)$ where

$$
F_{i}(x)=\frac{r_{i} x_{i}}{1+(A x)_{i}}, \quad r_{i}>1, A=\left(\left(a_{i j}\right)\right) \geq 0, a_{i i}>0, i, j=1, \ldots, d .
$$

There are a large number of papers that study the asymptotic dynamics of the LeslieGower model in various guises. However, here we are focussing on the carrying simplex, and so we restrict references to those that feature the carrying simplex. Jiang and Niu [24] studied heteroclinic orbits of the 3-dimensional Leslie-Gower model on the carrying simplex, and in [23] showed that the carrying simplex exists for the $d$-dimensional Leslie-Gower model, regardless of parameter values $r_{i}>1$ and $a_{i j}>0$ and used it, together with an index theorem, to identify 33 classes for orbits of th 3-dimensional Leslie Gower model.

Baigent [5] studied the existence of the planar Leslie-Gower model under similar conditions, and also showed that the carrying simplex was either a convex or concave curve. Recently, Baigent studied the 3-dimensional Leslie-Gower model [3] in 
May-Leonard form and established parameter regions where the carrying simplex was either convex or concave.

$F$ is continuously differentiable on $-\varepsilon(1, \ldots, 1)+C_{+}$for $\varepsilon>0$ small enough. The unique $d$ axial fixed points are positioned at $q_{i}=\frac{r_{i}-1}{a_{i i}}>0$. Hence AS1 and AS2 are satisfied. Here we have $f_{i}(x)=\frac{r_{i}}{1+(A x)_{i}}$ so that $f \gg 0$ on $C_{+}$, and

$$
(D f)_{i j}(x)=-\frac{r_{i} a_{i j}}{\left(1+(A x)_{i}\right)^{2}},
$$

so that $D f \ll 0$ on $C_{+}$. Hence $F$ satisfies AS3.

For AS4 we have $M(x)_{i j}=\frac{x_{i} a_{i j}}{1+(A x)_{i}}$ so that $M$ is a nonnegative matrix on $C_{+}$and $M(x) \gg 0$ for $x \in \operatorname{int} C_{+}$. Now we employ an elegant trick via lemma 18 as used by Jiang and Niu [23]. Take $D$ to be the diagonal matrix with diagonal entries $x$. Then $D^{-1} M(x) D$ has $i, j$ th element $\frac{x_{j} a_{i j}}{1+(A x)_{i}} \in(0,1)$. Hence using the max norm, or the infinity norm, we obtain that $\rho(M(x))<1$ and the result due to Jiang and Niu [23] that the carrying simplex exists for all $r \gg 0$ and $A \gg 0$. Figure 6 shows the evolution $F^{k}(\Delta)$ for $k=0,1,2,3,4$ for two sets of parameters. In (a) the iteration starts with $\Delta$ equal to the straight line segment going the two axial fixed points. From then the iteration $F^{k}(\Delta)$ is a decreasing sequence of curves that converges to the carrying simplex. Figure 4 (b) is similar, except $F^{k}(\Delta)$ is an increasing sequence of curves.
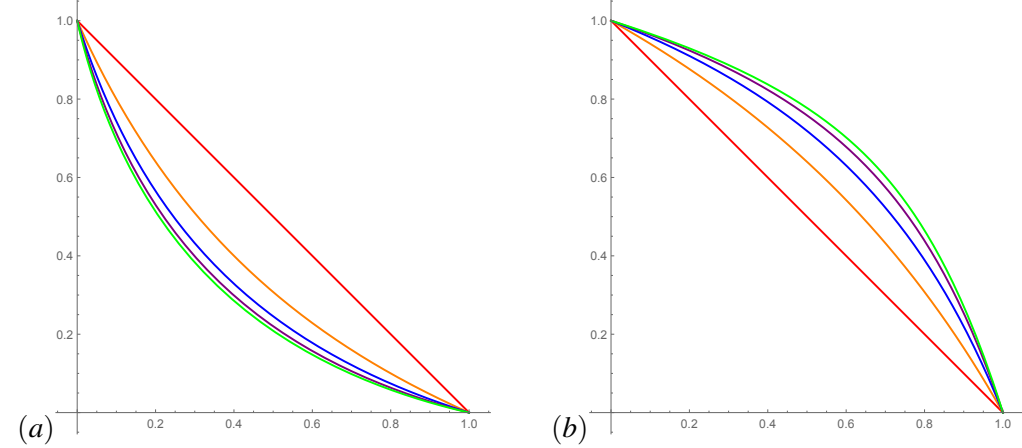

Fig. 6 Leslie-Gower model. Evolution of $F^{k}(L)$ for $k=0,1,2,3$, 4, where $L$ is the line joining $\left(r_{1}-1,0\right)$ and $\left(0, r_{2}-1\right)$. (a) $r_{1}=r_{2}=2, a_{12}=2, a_{21}=3$, (b) $r_{1}=r_{2}=2, a_{1,2}=a_{21}=1 / 2$.

Remark 9. Notice that the curves in each plot of Figure 4 are either convex or concave. From this it can be proved that the carrying simplex of the planar Leslie-Gower model is either convex or concave [5]. 


\subsection{The Ricker model}

The Ricker model takes the form

$$
F_{i}(x)=x_{i} \exp \left(r_{i}\left(1-(A x)_{i}\right)\right), A=\left(\left(a_{i j}\right)\right) \geq 0, a_{i i}=1, i=1, \ldots, d .
$$

The general model (9) has attracted considerable interest, especially in the past few years, mainly around conditions for global stability of the interior fixed point. In an early paper by Jiang and Rogers [21], the authors discuss the planar model $F(x, y)=\left(x e^{r(1-x)-s y)}, y e^{r(1-y)-s x)}\right)$. The derivative of this map has vanishing determinant along the hyperbolic curve $\Gamma$ of points in $\mathbb{R}_{+}^{2}$ such that $\left(r^{2}-s^{2}\right) x y-r(x+y)+1=0$. When $r>s$ there are two branches to $\Gamma$, which we call $\Gamma_{1}$ and $\Gamma_{2}$, with $\Gamma_{1}$ lying below $\Gamma_{2}$. When $r<s$ there is only a single branch to $\Gamma$ in $\mathbb{R}_{+}^{2}$. In the case $r>s$, Jiang and Rogers show that the map $F$ folds $R_{+}^{2}$ over along the curve $F\left(\Gamma_{1}\right)$ and that $F\left(\Gamma_{1}\right)$ is the boundary in $\mathbb{R}_{+}^{2}$ of $F\left(\mathbb{R}_{+}^{2}\right)$ (see Figure 7).

For the general planar Ricker model $((9)$ when $d=2)$, there have been a number of recent papers that explore the evolution of the critical curve under repeated applications of $F$. We recall that the critical curve $L C_{-1}$ is the set of singular points of $F$, so for our $C^{1}$ maps $L C_{-1}=\left\{x \in C_{+}: \operatorname{det} D F(x)=0\right\}$. In [7] Balreira, Elaydi and Luís studied the planar Ricker map $F(x, y)=\left(x e^{r-x-a y}, y e^{s-y-b x}\right)$ for $a b<1$ and $r, s \in(1,2)$, addressing the problem: When does local asymptotic stability of an interior fixed point imply that it is globally asymptotically stable? Central to their work is the existence of an invariant manifold $\Sigma$ which is the union of the unstable manifolds for each axial fixed point.They considered successive images $F^{k}\left(L C_{-1}^{1}\right)$ of the lower branch $L C_{-1}^{1}$ of the critical curve $L C_{-1}=\left\{(x, y) \in \mathbb{R}_{+}^{2}\right.$ : $x+y-1-(1-a b) x y=0\}$ of singular points of $F$ (see [10]). Similar to Jiang and Rogers they found that $F\left(\mathbb{R}_{+}^{2}\right)$ is the region in $\mathbb{R}_{+}^{2}$ below $L C_{0}^{1}=F\left(L C_{-1}^{1}\right)$. Assuming firstly that $L C_{-1}^{1}$ lies below the two nullclines of $F$, and secondly that none of these images $F^{k}\left(L C_{-1}^{1}\right)$ intersected, they showed that $F^{k}\left(L C_{-1}^{1}\right) \rightarrow \Sigma$ as $k \rightarrow \infty$, and an interior fixed point was globally asymptotically stable. The manifold $\Sigma$ is the boundary in $\mathbb{R}_{+}^{2}$ of the basin of repulsion of the origin. When $F$ satisfies AS1-AS4 of Theorem, $\Sigma$ is the carrying simplex.

Ryals and Sacker [31] (see also [32]) consider the same Ricker model as in [7] and give sufficient conditions for the images $L C_{k-1}^{1}:=F^{k}\left(L C_{-1}^{1}\right)$ for $k=0,1, \ldots$ not to intersect, namely that $L C_{-1}^{1}$ lies below $F^{2}\left(L C_{-1}^{1}\right)$ which in turn lies below $F\left(L C_{-1}^{1}\right)$ and also that $F\left(L C_{-1}^{1}\right)$ does not intersect the upper branch of the critical curve. They also showed that both $L C_{-1}^{1}$ and $F\left(L C_{-1}^{1}\right)$ are the graphs of decreasing concave functions, but that in many cases the higher iterates lost monotonicity, concavity, may self-intersect and even fail to be the graph of a function (see example (d) in Figure 8 below)

The lower branch of the critical curve, $L C_{-1}^{1}$, is decreasing, concave and passes through $(1,0)$ and $(0,1)$, whenever $a>0, b>0$ and $a b<1$. Let $R_{1}=\left\{(x, y) \in \mathbb{R}_{+}^{2}\right.$ :

$x+y-1-(1-a b) x y>0\}$, that is the region in $\mathbb{R}_{+}^{2}$ below the critical curve $L C_{-1}^{1}$ (where $\operatorname{det} D F>0$ ). $F\left(\mathbb{R}_{+}^{2}\right)$ is the region in $\mathbb{R}_{+}^{2}$ below $L C_{0}^{1}=F\left(L C_{-1}^{1}\right)$. Moreover, 


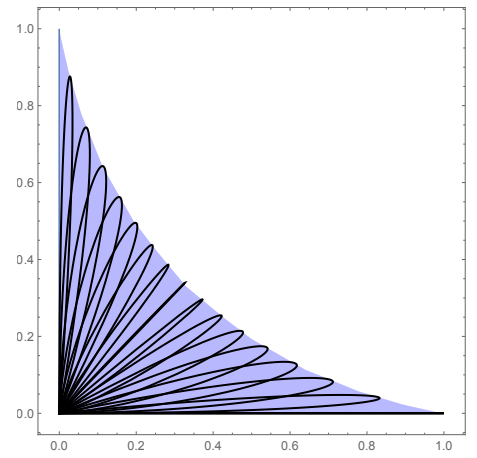

Fig. 7 The planar Ricker model. Image of $\mathbb{R}_{+}^{2}$ under $F$ when $r_{1}=r_{2}=1, a_{12}=a_{21}=2$. Each loop is $\left\{F(t u): t \in \mathbb{R}_{+}\right\}$for some $u$ in the unit probability simplex. The union of all the loops is $F\left(\mathbb{R}_{+}^{2}\right)$ and is the shade region. Notice that the loops indicate folding over of $\mathbb{R}_{+}^{2}$ along the boundary of the shaded region.

$D F=\left(\begin{array}{cc}(1-x) e^{r-x-a y} & -a x e^{r-x-a y} \\ -b y e^{s-y-b x} & (1-y) e^{s-y-b x}\end{array}\right)$ so that $D F^{-1}(x, y) \gg 0$ for $(x, y) \in R_{1}$. In particular, when $r, s \in(0,1), F\left(\mathbb{R}_{+}^{2}\right) \subseteq R_{1}, F$ is competitive has a carrying simplex. While $F$ is no longer competitive if either $r>1$ or $s>1$, the example (b) of Figure 8 shows that there still may be a unordered invariant manifold that attracts $\mathbb{R}_{+}^{2} \backslash\{0\}$.

For the higher dimensional Ricker model (9), Gyllenberg, Jiang, Niu and Yan [15] recently found sufficient conditions for the existence of a carrying simplex (see (10) and (11) below) although this is not the focus of their paper. In both cases it is necessary that $r_{i}<1$ for $i=1, \ldots, d$. To obtain these conditions, note that the Ricker map $F(9)$ has axial fixed points at $q_{i}=\left(a_{i i}^{-1}\right) e_{i}$ for $i=1, \ldots, d$. We need to establish that $\rho(D[r] D[x] A)<1$ for $x \in \Lambda$. Now by the ordering property of the spectral radius $\rho(D[r] D[x] A) \leq \rho(D[r] D[q] A)$ for all $x \in \Lambda$, and so we need only find conditions that ensure that $\rho(D[r] D[q] A)<1$. From $\rho(M(q)) \leq\|M(q)\|_{\infty}$ we obtain

$$
\|M(q)\|_{\infty}=\max _{1 \leq i \leq d} \sum_{j=1}^{d}-\frac{q_{i}}{f_{i}(q)} \frac{\partial f_{i}}{\partial x_{j}}(q)=\max _{1 \leq i \leq d} \frac{1}{a_{i i}} \sum_{j=1}^{d} r_{i} a_{i j}
$$

which is less than one if

$$
r_{i}<\frac{a_{i i}}{\sum_{i=1}^{d} a_{i j}}, i \in I_{d}
$$

and also we have (using the same trick as in the Leslie-Gower model)

$$
\|M(q)\|_{\infty}=\max _{1 \leq i \leq d} \sum_{j=1}^{d}-\frac{q_{j}}{f_{i}(q)} \frac{\partial f_{i}}{\partial x_{j}}(q)=\max _{1 \leq i \leq d} \sum_{j=1}^{d} r_{i} \frac{a_{i j}}{a_{j j}}
$$

which is less than one if 

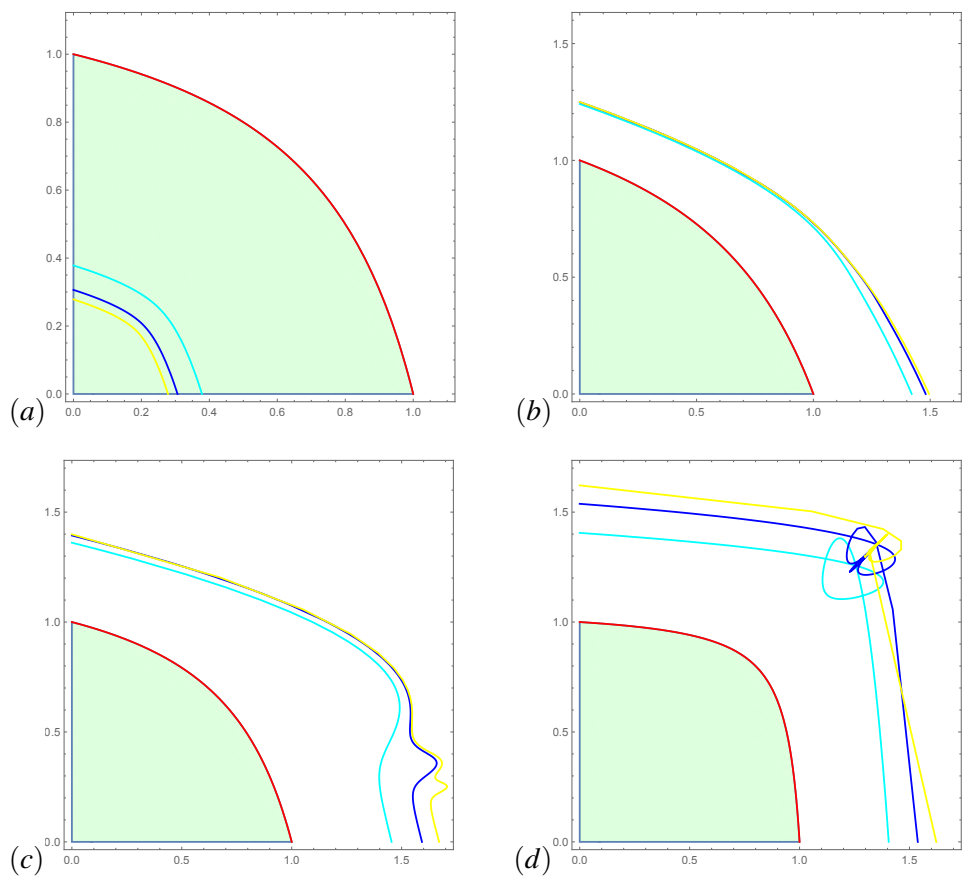

Fig. 8 The planar Ricker model. The shaded area is where $F$ is competitive. (a) $a=b=0.5, r=$ $s=0.25$, (b) $a=0.25, b=0.75, r=1.5, s=1.25$, (c) $a=0.35, b=0.75, r=1.8, s=1.4$, (d) $a=b=0.25, r=s=1.9$. In (a), (b) there is a carrying simplex by Hirsch's definition.

$$
r_{i}<\frac{1}{\sum_{j=1}^{d} \frac{a_{i j}}{a_{j j}}}, i \in I_{d}
$$

The two conditions (10) and (11) are those conditions obtained in [15].

\section{Discussion}

The carrying simplex is an interesting example of a compact invariant manifold of codimension-one that is globally attracting bar the origin. We showed that a competitive map transforms unordered hypersurfaces into unordered hypersurfaces, and that these unordered hypersurfaces are all graphs of Lispchitz functions. In this way we build the usually required equicontinuity into our existence proof. We could also have used the Schauder fixed point theorem to obtain existence of a Lipschitz invariant hypersurface (as was done in [5]). There are thus a good number of similarities between the existence proof for a carrying simplex offered here and proofs of stable and unstable manifolds using cone fields (e.g. [25]), or inertial manifolds [29]. How- 
ever, many of these proofs apply the contraction principle, and thus obtain global attraction, and some smoothness, for free. Here we do not explicitly use a contraction principle, so that we have to work a little harder to obtain global attraction, and this was done by way of a measure $\mu$ on phase space that strictly decreases under $F$. As a result, we do not obtain smoothness for free, and at present the problem of smoothness is partially open. Necessary and sufficient conditions for the smoothness of the (entire) carrying simplex are known [22,27], but to the best of the author's knowledge refined conditions are not yet available (in dimension $d>2$ ) for when the carrying simplex is smooth on its interior and not necessarily at boundary points.

Before finishing, we mention some future directions of research regarding carrying simplices: Establish

- conditions for $C^{r}$ smoothness of the interior of the carrying simplex.

- carrying simplex existence results for general convex cones (i.e. beyond the standard positive cone).

- the relations between carrying simplex geometry and stability of fixed points and the existence of periodic orbits. Some progress has been made here for continuous time competitive Lotka-Volterra systems by Zeeman and Zeeman [40]. Recent progress on the convexity or concavity of carrying simplices for competitive maps can be found in $[5,3]$.

- a bifurcation analysis that links change in stability to change in geometry of the carrying simplex local to a fixed point.

- how the carrying simplex can be utilised to obtain a global picture of dynamics. For recent progress in this direction see [28, 23, 15].

- how the carrying simplex might be used to show existence or nonexistence of periodic orbits.

Acknowledgements I would like to thank the organisers of 23rd International Conference on Difference Equations and Applications (ICDEA 2017) in Timişoara, Romiana for their hospitality and the opportunity to contribute to the conference proceedings.

\section{References}

1. Ambrosetti, A., Prodi, G. A primer of nonlinear analysis. No. 34. Cambridge University Press, 1995.

2. Bhatia, N. P., Szegö, G. P. Stability Theory of Dynamical Systems. Springer Science \& Business Media, 2002.

3. Baigent, S. (2018). Convex geometry of the carrying simplex for the May-Leonard map. Discrete and Continuous Dynamical Systems Series B, 24(4):1697-1723.

4. Baigent, S. (2013). Geometry of carrying simplices of 3-species competitive Lotka-Volterra systems. Nonlinearity, 26(4):1001-1029.

5. Baigent, S. (2016). Convexity of the carrying simplex for discrete-time planar competitive Kolmogorov systems. Journal of Difference Equations and Applications, 22(5):1-14.

6. Baigent, S. (2012). Convexity-preserving flows of totally competitive planar Lotka-Volterra equations and the geometry of the carrying simplex. Proceedings of the Edinburgh Mathematical Society, 55:53-63. 
7. Balreira, E. C., Elaydi, S., Luís, R. (2014). Local stability implies global stability for the planar Ricker competition model. Discrete and Continuous Dynamical Systems Series B, 19(2):323-351.

8. Balreira, E. C., Elaydi, S., Luís, R. (2017). Global stability of higher dimensional monotone map. Journal of Difference Equations and Applications, 23(12):2037-2071.

9. Berman, A., Plemmons, R. J. Nonnegative matrices in the mathematical sciences Vol 9, SIAM, 1994.

10. Cathala, J. C. (1983). Absorptive area and chaotic area in two-dimensional endomorphisms. Nonlinear Analysis, Theory, Methods and Applications, 7(2):147-160.

11. Cushing, J. M., Levarge, S., Chitnis, N., Henson, S. M. (2004). Some Discrete Competition Models and the Competitive Exclusion Principle. Journal of Difference Equations and Applications, 10(13-15):1139-1151.

12. de Mottoni, P., Schiaffino, A. (1981). Competition Systems with Periodic Coefficients: A Geometric Approach. Journal of Mathematical Biology, 11:319-335.

13. Diekmann, O.,Wang, Y., Yan, P. (2008). Carrying Simplices in Discrete Competitive Systems and Age-structured Semelparous Populations. Discrete and Continuous Dynamical Systems Series B, 20(1):37-52.

14. Gale, D., Nikaidô, H. (1965) The Jacobian Matrix and Global Univalence of Mappings. Mathematische Annalen, 159: 81-93.

15. Gyllenberg, M., Jiang, J., Niu, L., Yan, P. (2017). On the dynamics of multi-species Ricker models admitting a carrying simplex. (Preprint).

16. Hirsch, M. W., Smith, H. (2005), Monotone maps: a review, Journal of Difference Equations and Applications, 11(4-5):379-398.

17. Hirsch, M. W., Smith, H. Monotone Dynamical Systems, In Handbook of differential equations: ordinary differential equations, vol. 2, 239-357 North-Holland, 2006.

18. Hirsch, M. W. (1988). Systems of differential equations which are competitive or cooperative: III Competing species. Nonlinearity, 1:51-71.

19. Hirsch, M. W. (2008). On existence and uniqueness of the carrying simplex for competitive dynamical systems. Journal of Biological Dynamics, 2(2):169-179.

20. Horn, R. A., Johnson, R. J. Topics in Matrix Analysis, Cambridge University Press, 1994.

21. Jiang, H., Rogers, T. D. (1987). The discrete dynamics of symmetric competition in the plane. Journal of Mathematical Biology, 25:1-24.

22. Jiang, J., Mierczyński, J., Wang, Y. (2009). Smoothness of the carrying simplex for discretetime competitive dynamical systems: A characterization of neat embedding. Journal of Differential Equations, 246(4):1623-1672.

23. Jiang, J., Niu, L. (2016). On the equivalent classification of three-dimensional competitive Leslie-Gower models via the boundary dynamics on the carrying simplex. Journal of Mathematical Biology, 74(5):1-39.

24. Jiang, J., Niu, L., Wang, Y. (2015). On heteroclinic cycles of competitive maps via carrying simplices. Journal of Mathematical Biology, 72(4):939-972.

25. Katok, A., Hasselblatt, B. Introduction to the modern theory of dynamical systems. Cambridge University Press, 1995.

26. McShane, E. J. (1934). Extension of range of functions. Bulletin of the American Mathematical Society, 40(12):837-843.

27. Mierczyński, J. (2018). The $C^{1}$ property of convex carrying simplices for three-dimensional competitive maps. Journal of Difference Equations and Applications, 55:1-11.

28. Niu, L., Jiang, J. (2016). On the equivalent classification of three-dimensional competitive Atkinson/Allen models relative to the boundary fixed points. Discrete and Continuous Dynamical Systems Series B, 36(1):217-244.

29. Robinson, J. C. (1993). Inertial manifolds and the cone condition. Dynamic Systems and Applications 2:311-330.

30. Ruiz-Herrera, A. (2013). Exclusion and dominance in discrete population models via the carrying simplex. Journal of Difference Equations and Applications, 19(1):96-113.

31. Ryals, B., Sacker, R. J. (2015). Global stability in the 2D Ricker equation. Journal of Difference Equations and Applications, 21(11):1068-1081. 
32. Ryals, B., Sacker., R. J. (2016). Global stability in the 2D Ricker equation revisited. Discrete and Continuous Dynamical Systems Ser B, 22(2):585-604.

33. Smith, H. L. (1986) Periodic competitive differential equations and the discrete dynamics of competitive maps. Journal of Differential Equations, 64(2):165-194.

34. Smith, H. L. (1986) Periodic solutions of periodic competitive and cooperative systems. SIAM Journal on Mathematical Analysis, 17(6):1289-1318.

35. Smith, H. L. (1998) Planar competitive and cooperative difference equations. Journal of Difference Equations and Applications, 3(5-6):335-357.

36. Smith, H. L. (1986) Periodic orbits of competitive and cooperative systems. Journal of Differential Equations, 65:361-373.

37. Takáč, P. (1990). Convergence to equilibrium on invariant d-hypersurfaces for strongly increasing discrete-time semigroups. Journal of Mathematical Analysis and Applications, 148(1):223-244.

38. Wang, Y. Jiang, J. (2001) The General Properties of Discrete-Time Competitive Dynamical Systems Journal of Differential Equations, 176(2): 470-493.

39. Wang, Y., Jiang, J. (2002). Uniqueness and attractivity of the carrying simplex for discretetime competitive dynamical systems. Journal of Differential Equations, 186(2):611-632.

40. Zeeman E. C., Zeeman, M. L. (2003). From local to global behavior in competitive LotkaVolterra systems. Transactions of the American Mathematical Society, 355(2):713-734.

41. Zeeman, M. L. (1993). Hopf bifurcations in competitive three-dimensional Lotka-Volterra systems. Dynamics and stability of systems 8(3):189-216. 